\title{
IPR Protection in the High-Tech Industries: A Model of Piracy
}

Thierry Rayna

Discussion Paper No. 06/593

August 2006

Department of Economics

University of Bristol

8 Woodland Road

Bristol BS8 1TN 


\title{
IPR Protection in the High-Tech Industries: A Model of Piracy
}

\author{
Thierry Rayna*
}

August 2006

\begin{abstract}
This article investigates the relation between the level of publicness of digital goods i.e. their degree of non-excludability and non-rivalness - and the pirating behaviour of the consumers. The main focus is put on the difference between the ex-ante level of publicness - determined by the anti-piracy strategies of the firms - and the ex-post level of publicness - which is a consequence of external factors such as the consumers network structure, the consumers sharing behaviour, etc. The two models developed in the article detail the required conditions for anti-piracy strategies to be successful and show the influence of the economic environment on these conditions.

Keywords:

Digital goods, Piracy, Public goods, Free-riding, Intellectual Property Rights

JEL Codes:

D11, C72, L82, L86, O34
\end{abstract}

\section{Introduction}

The industry of digital goods (music, movies, documents, software, etc.) stands among the most innovative industries. However, the growth and viability of the companies in this industry are seriously undermined by the extent of consumers piracy, which seems to be - in addition to innovation - the main characteristics of this sector. It is therefore of the first importance to understand the reasons behind such a widespread piracy phenomenon, and the factors that influence it, in order to be able to control it.

We think that the reason for which so many consumers adopt a piracy behaviour lies in the fact that digital goods have common characteristics with public goods. Indeed, since digital goods can be easily cloned - i.e. copied perfectly - they tend to be both non-rival an non-excludable. Thus the piracy behaviour of consumers is nothing else but the rational individual behaviour stated in the literature: when consumers are asked to contribute to the provision of a public good, the rational behaviour is to free-ride and not to contribute at all. Empirical studies show that in the case of digital goods, this rational behaviour has indeed been adopted by lots of consumers.

*Address: Department of Economics, University of Bristol, 8 Woodland Road, Bristol BS8 1TN, United Kingdom. Email Address: thierry.rayna@bristol.ac.uk. 
However, the publicness of digital goods - e.g. the degree of non-excludability and non-rivalness of these goods - is not always total. In addition to the obvious role of technology, other factors influence the degree of publicness of the digital goods: the connectivity of the consumers network, the behaviour of the consumers, the laws and public policies. Last but not least, the actions of the firms plays a determinant role. Indeed, the anti-piracy strategies adopted by the firms have an important impact on the degree of publicness of the digital goods.

Therefore the extent of the anti-piracy effort of the firms determines, for a given technology, the ex-ante level of publicness of the digital goods. However, other factors, such as the structure of the consumers' networks, the behaviour of the consumers and their ability to avoid or disable anti-piracy protections, change the initial publicness level and lead to an ex-post level of publicness. This ex-post level of publicness will, in turn, influence the piracy behaviour of the consumers: a high level of publicness will lead to a high level of piracy whereas a low level of publicness will push the consumers to purchase the good instead.

The aim of this article is to build a model analysing the piracy and the sharing behaviour of the consumers, based on the factors influencing both the ex-ante and ex-post publicness of the digital goods.

After discussing briefly the publicness of digital goods in Section 1, Section 2 introduces the model. Section 3 looks at the individual behaviour of consumers and presents the pre-conditions that are required for them to pirate and share. Section 4, investigates the issue of pirating and sharing in a non-repeated game. Building on the results of this previous section, Section 5 examines the conditions required for a cooperative equilibrium to exist in an infinitely repeated game. Finally, based on the results obtained in the previous sections, Section 6 analyses the anti-piracy strategies of the firms and discusses the issue of network structure, anonymity and cooperation.

\section{The publicness of digital goods}

Digital goods are goods that are distributed in a digital format - i.e. encoded in binary form, as a succession of $0 \mathrm{~s}$ and $1 \mathrm{~s}$. Due to this digital nature, digital goods are independent from the medium used to distribute them since the binary form used to encode them ensures that these goods can be transferred from one medium to another without loss of quality or information. Thus digital goods are "clonable": any digital good can be duplicated (on the same type of medium, or any medium able to store digital data), and the duplicate of the digital good is then the exact replica - a clone - of the digital good itself.

As digital goods can be cloned easily and for a very low cost, they can be considered as non-rival: several consumers can consume the same unit of digital good simultaneously, provided that each of the consumers made a copy of the digital good. Ultimately, only one unit of the digital good has to be purchased for all the consumers to enjoy simultaneously the digital good. Likewise, the ability of cloning digital goods make them also non-excludable, since the producers, once the first unit of the good was sold, are not able to exclude consumers from the consumption of the good since consumers can copy the good from each other. Therefore, the digital nature of digital goods make them non-rival and 
non-excudable, and as such, we can consider that digital goods are public goods.

We can therefore expect digital goods, as any other public good, to be subject to free-riding behaviour. And this is indeed the case: the high degree of adoption of the piracy behaviour among the consumers can be explained by the fact that pirating a digital good is in fact the strict equivalent to free-riding. Instead of buying the digital goods, consumers prefer to wait for somebody else to contribute/buy the digital good, as they can subsequently copy the digital good from this person.

Thus we can consider that piracy is actually based on a rational behaviour: pirating digital goods is in fact free-riding. Leaving aside the questions of ethics and moral, it is difficult to blame the consumers for adopting such a behaviour since this is, according to the theory, the adequate individual rational behaviour when facing a public good. From this point of view, the problem of piracy is not caused by the consumers but is instead due to the nature of digital goods: if these goods were private, the piracy phenomenon would not exist.

However, the publicness of digital goods depends on a several factors and is not always total ${ }^{1}$. Indeed, the factors that influence the level of rivalness and excludability of digital goods $\operatorname{are}^{2}$ :

- The available technology

- The structure of the consumers' network

- The behaviour/strategies of the consumers

- The strategies of the firms

- The policies of the governments

As all these factors evolve over time, the levels of rivalness and excludability - and hence the level of publicness of digital goods - also evolve over time. Therefore, free-riding/pirating is not necessarily the only rational behaviour for consumers: if the publicness for a particular digital good is low, consumers are more likely to buy it instead.

It is thus common for the firms producing digital goods to adopt anti-piracy strategies aiming at decreasing the publicness of digital goods. By doing so, they decrease the likelihood of consumers pirating. Rayna (2002) shows that all the strategies the firms can adopt to fight against piracy consist in either an increase of excludability or an increase of rivalness - or both - of the digital good. DRM, serial numbers or Microsoft's Windows Product Activation, for example, aim at increasing the excludability of the digital good, since they - theoretically prevent consumers who did not obtain the digital good legitimately from using it. On the contrary, dongles, compulsory use of the original medium (floppy disk, CD, DVD), network scans for identical serial numbers, etc. aim at increasing the rivalness since they ensure that only one unit of the digital good is being used at the same time ${ }^{3}$.

\footnotetext{
${ }^{1}$ See Rayna (2002) for a detailed discussion of this topic.

2 The structure of consumers' network, the behaviour of the consumers and the strategies of the firms are considered explicitly in the models developed in the next sections. The available technology is implicitly taken into in the cost of searching and copying, as well as in the network connectivity. The influence of public policies is discussed in section 6 .

${ }^{3}$ These technologies do not however verify that the unit of digital good being used is used by a legitimate user. Thus they do not increase - directly - the excludability.
} 
As an intrinsic property of the digital good - anti-piracy systems are most of the time embedded in the software, music file, etc. - the anti-piracy strategies of the firms determine the ex-ante level of publicness. However, the other determinants of the publicness, which are all external factors, also play an important role, and lead to what we define as ex-post publicness. For example, it is possible that the firms do not adopt any anti-piracy behaviour. In this case, the ex-ante publicness of the digital good is total. Nevertheless, if consumers are not connected to any network, if copying the digital good is very costly, or if consumers never share their digital goods, the ex-post publicness of the digital good is null since in any of these cases the producers remains the sole supplier of the good.

On the contrary, it is possible that the firms design a - theoretically - perfect anti-piracy system such that the ex-ante publicness is null. However, if a program allowing to remove the anti-piracy system from the digital good is available for the consumers, or if one of the consumers got hold of a protectionfree version of the digital good, the ex-post publicness may be total in spite of an initial null ex-ante publicness.

More interestingly, it is possible that the external determinants of publicness are such that a positive ex-ante publicness will lead to a to a null ex-post publicness - e.g. depending on the external determinant, it is possible that the anti-piracy systems designed by the firms, although not perfect, may be "annoying" enough to deter consumers from pirating.

This is precisely this type of problems that the models developed in the next sections aim at analysing.

\section{Description of the model}

In this model, we assume a homogeneous population of consumers. There is only one homogeneous digital good available for their consumption. The digital good is "short-lived" as the consumers do not wish to consume the same digital good for more than one period. A new homogeneous digital good is produced at the beginning of each period.

The goal pursued by each consumer is to consume exactly one unit of digital good per period. At the beginning of each period, the consumers have the choice between purchasing the digital good from the producer and pirating the good. If they decide to purchase the good from the producer they obtain the good and their payoff corresponds to the utility brought by the consumption of the digital good minus the cost of purchasing the good (i.e. the market price of the digital good).

On the other hand, if the consumers decide to pirate the good - i.e. to obtain a copy of the digital good through pirate channels ${ }^{4}$ - their access to the good becomes uncertain. A few conditions are indeed required for a piracy decision to be successful. First, the consumer must part of a network of consumers who already own the digital good. If the consumer is not connected to any network, or if none of the consumers on the network possesses the digital good,

\footnotetext{
${ }^{4}$ The main focus of this model is put on the online piracy, we therefore assume that the consumers pirate the digital good by downloading it. Nevertheless, this model could also describe - with minor changes - "hand-to-hand" offline piracy, and black market piracy. Most of the results of this model can in fact be generalised to other forms of piracy.
} 
the choice of piracy will lead to a failure since the consumer will not be able to obtain a copy of the digital good. Assuming that the consumer is able to find on the network another consumer who owns a copy of the the digital good, she then has to convince this consumer to let her copy the digital good. If the consumer refuses to share the digital good, the piracy behaviour will be, once again, unsuccessful. If the consumer accepts to share the digital good, the first stage of the piracy behaviour is successful and the consumer is able to make a copy of the digital good ${ }^{5} 6$.

However, the firms supplying the digital goods are usually not passive in regards to piracy and try to prevent consumers from pirating the digital goods. Thus, once the consumer obtains a pirated copy of the digital good, she can actually enjoy the consumption of the digital good only if neither exclusion nor rivalness take place. If either exclusion or rivalness take place, the pirating consumer is unable to consume the good and gains no utility from possessing a copy of the digital good while still bearing the costs associated with pirating the digital good - i.e. the search cost and the cost of copying ${ }^{7}$. What is more, if rivalness takes place, the consumer who shared the digital good with the pirating consumer also looses the ability to consume the digital good and is thus left with a loss equivalent to the utility this good has for her.

\section{$2.1 \quad$ Timeline}

We assume that each consumer plays once per period:

1. The consumer chooses between purchasing and pirating the digital good. If the consumer chooses to purchase the good, she pays the price for this good and obtains the utility associated with the consumption of the good. The consumer then waits for the next period.

2. If the consumer decides to pirate, she has to find somebody on the network who owns a copy of the digital good. If the network is not sufficiently connected, the consumer may not find a source for digital good. In this case, the consumer does not get any utility, as no digital good is consumed, but still has to bear the cost of searching for the good on the network.

3. If the consumer is able to find a source - i.e. another consumer who owns a copy of the digital good - she obtains a copy of the digital good only if the "source" consumer allows for the digital good to be copied. If this is not the case, the consumer does not get any utility, as no digital good is consumed, but still has to bear the cost of searching for the good.

4. If the consumer manages to obtain a copy of the digital good, she can consume it if and only if the original producer of the digital good is not able to exclude from the consumption of the good the consumers who

\footnotetext{
${ }^{5}$ We assume, as it is usually the case, that the cost of obtaining a pirated copy of the digital good, including the cost of the copy and the opportunity cost of searching and copying the good, is lower than the cost of purchasing a legitimate copy of the digital good.

${ }^{6}$ We assume that the quality of the digital good is the same regardless of its origin - bought or pirated - and thus gives the same level of utility to the consumer.

${ }^{7}$ It is worth noticing that firms are, in general, not able to destroy the pirated copy of the good. Thus despite the fact that the consumer is unable to use the digital copy, this copy can still be used a source of copies for other consumers trying to pirate the good.
} 
did not pay for it. If the producer can exclude the consumer from the consumption of the good, the consumer does not get any utility, as no digital good is consumed, but still has to bear the cost of searching for the good and copying the digital good.

5. If the consumer is not excluded from the consumption of the good, she can still be prevented form consuming the good if the producer is able to introduce some rivalness in the consumption of the good. In this case, the consumer is able to consume the good if and only if no other consumer who made a copy from the same original unit of digital good (including the consumer owning the original unit of the digital good) is consuming the good at the same time. Thus, as the number of consumers pirating grows, the probability that any of the consumers is able to consume the digital good decreases. Ultimately, it is impossible to consume the digital good when there is rivalness, and the consumer does not get any utility, as no digital good is consumed, but still has to bear the cost of searching for the good and copying the digital $\operatorname{good}^{8}$.

6. If there is no rivalness, the consumer consumes the pirated digital good and obtains the utility associated with this consumption. In this case the cost borne by the consumer is the cost of searching and copying the digital good. The consumer then waits for the next period.

\subsection{The environment}

The environment in which the consumers evolve is described by the following variables:

The network : $N_{i} \in[0,1]$ describes the connectivity of the network for the consumer $i$. This is the probability that consumer $i$ will find a source for the digital good once she has decided to pirate the good. If $N_{i}=0$ the consumer is not connected to any other consumer and any attempt to pirate will be unsuccessful. If $N_{i}=1$ the consumer who decides to pirate will always find another consumer who owns a copy of the digital good.

Excludability : $E \in[0,1]$ represents the level of excludability of the digital good. This is the probability that the producer will be able to exclude a consumer who obtained a pirated copy of the good. If $E=1$, the excludability is total and no consumer who did not legitimately purchase the digital good is able to consume it. If $E=0$, the digital good is non-excludable and the producers are unable to monitor and prevent illegitimate users from consuming the good.

Rivalness : $R \in[0,1]$ represents the level of rivalness of the digital good. If $R=1$, the rivalness is total and only one unit/copy of the digital good can be used at the same time. Practically, as the number of copies becomes large, the consumers are prevented from using the digital good, including the legitimate owner of the digital good as long as they let other consumers copy their unit of digital good. If $R=0$, the digital good is non-rival,

\footnotetext{
${ }^{8}$ Likewise, the consumer who shared the good is also prevented from consuming the good and faces a loss equal to the utility brought by the consumption of the digital good.
} 


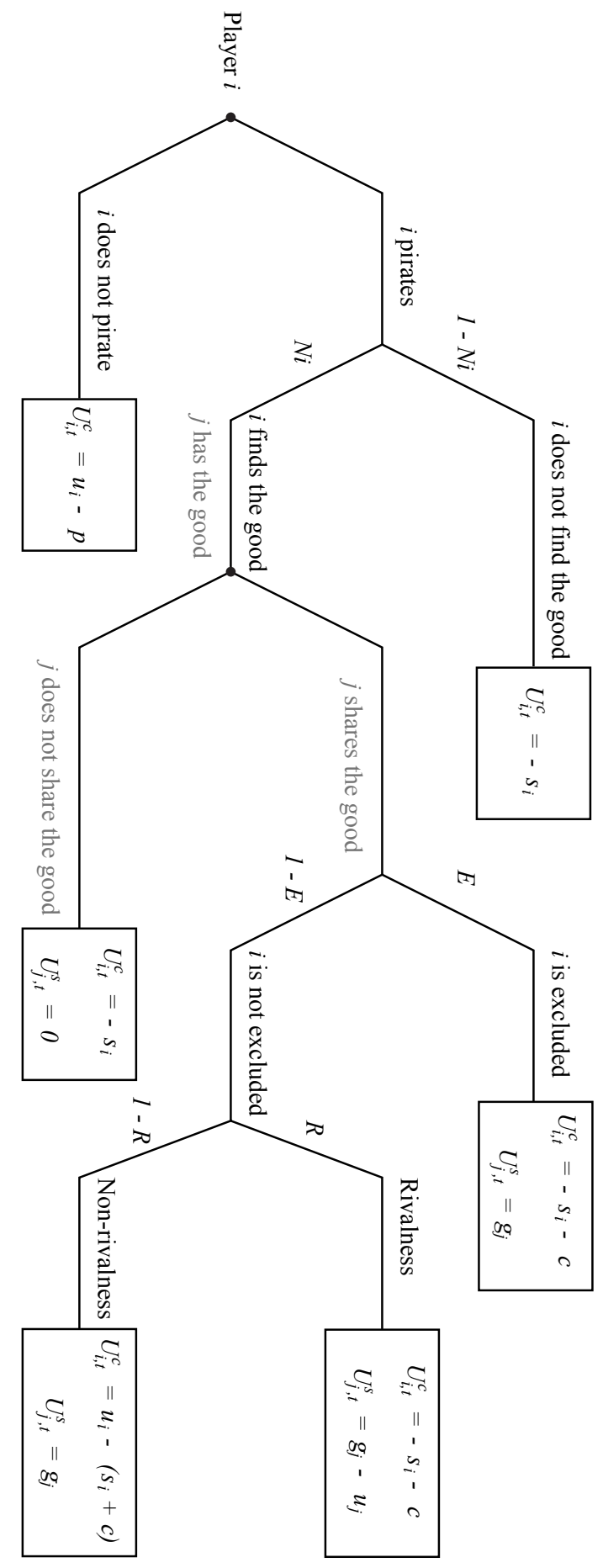

Figure 1: Individual game tree 
and an infinite number of consumers can consume simultaneously copies of the same original unit of digital good.

\subsection{The payoffs}

If the consumer is able to obtain a unit of digital good (legitimate or pirate) and is able to consume it, she receives a utility $u_{i}{ }^{9}$; she receives 0 otherwise. In order to calculate the consumers' payoffs, the cost incurred are deducted from the utility obtained by the consumer:

- The price $p$ if the consumer purchases the good from the producer.

- The search/opportunity cost $s$ if the the consumer attempts to find the good on the network.

- The copying cost $c$ if the consumer makes a copy of the digital good.

Thus the payoff brought by the consumption activity of the consumer $i$ during period $t, U_{i, t}^{c}$ is:

- $U_{i, t}^{c}=u_{i}-p$ if the consumer purchases the good.

- $U_{i, t}^{c}=-s$ if the consumer decides to pirate the good but did not find a source.

- $U_{i, t}^{c}=-s-c$ if the consumer pirates the good but is not able to consume it due to rivalness or excludability.

- $U_{i, t}^{c}=u_{i}-(s+c)$ if the consumer pirates the good and is able to consume it.

In addition, each consumer can be contacted by another consumer willing to make a pirate copy of the digital good she owns. If consumer $i$ accepts to let another consumer make a copy of a digital good, she gets a reward $g_{i}$ - positive or negative. However, if a consumer lets another consumer copy her own unit of digital good and if rivalness occurs ${ }^{10}$, she is not able to consume her unit of digital good anymore. Thus her payoff decreases by $u_{i}$. So if a consumer $i$ is contacted by another consumer $j \neq i$ asking her to copy the digital good, she obtains the following sharing payoff, $U_{i, t}^{s}$ :

- $U_{i, t}^{s}=0$ if she refuses to share the good and does not let the other consumer copy it.

- $U_{i, t}^{s}=g_{i}$ if she accepts to let the other consumer make a copy and rivalness does not take place.

- $U_{i, t}^{s}=g_{i}-u_{i}$ if she accepts to let the other consumer make a copy and rivalness occurs

\footnotetext{
${ }^{9}$ We assume that the quality of the digital good is the same regardless of its origin. However, a difference of quality could be taken into account in the price/costs of the digital goods.

${ }^{10}$ We assume here that the consumer who is used as a source for the copy of the digital good is left unaffected by excludability, if excludability occurs after a consumer copied the digital good from her. This is either due to the fact that she is a legitimate user, or because the imperfect monitoring of the firms only allow them to exclude some illegitimate users.
} 
The payoff of a consumer $i$ for a period $t, U_{i, t}$ is the sum of the payoffs obtained due to the consumption and, possibly, the sharing of the digital good.

$$
U_{i, t}=U_{i, t}^{c}+U_{i, t}^{s}
$$

\subsection{Assumptions on the payoffs}

We assume that the consumer would be better off pirating the good rather than buying it. Thus, by assuming that the quality and the characteristics of the digital goods are the same regardless of their origin, we assume that the price that the consumer would have to pay to purchase the good is higher than the cost of pirating the good:

$$
p>s_{i}+c
$$

Thus:

$$
u_{i}-p<u_{i}-\left(s_{i}+c\right)
$$

In order to discuss this problem thoroughly, no prior assumptions are made about the value of $g_{i}$, which is a combination of the costs and rewards of sharing the good. Depending on the situation, $g_{i}$ can be positive, negative or null. All three cases will be discussed in the next sections.

\section{Individual Behaviour}

\subsection{Sharing or not sharing?}

The first element to determine is the consumers' willingness to share. Indeed, regardless of the nature of the digital goods, and of the behaviour of the firms, piracy can not take place if none of the consumers is sharing: in this case the good is fully excludable as the producer is the sole supplier/source of digital good.

The game and the payoffs faced by a consumer asked to share are described in Figure 2. By combining the outcomes linked to independent events (exclusion and rivalness) leading to the same payoff, the game can be simplified as in Figure 3.

Looking at Figure 3, it becomes clear that the decision whether to share the digital good or not depends on the difference between the "Not sharing" payoff (0) and the expected value of the sharing payoff:

$$
(1-E) R\left(g_{i}-u_{i}\right)+1-(1-E) R g_{i} \equiv g_{i}-(1-E) R u_{i}
$$

As the payoff when the consumer does not share is zero, the consumer will agree to share the good in a single stage game if and only if the payoff of sharing is greater than zero:

$$
g_{i}-(1-E) R u_{i} \geq 0 \Leftrightarrow g_{i} \geq(1-E) R u_{i}
$$

Provided that $E$ and $R$ are probabilities, and $u_{i} \geq 0$, a necessary condition for the consumer to share is $g_{i} \geq 0$. These results are summarised in Proposition 1. 


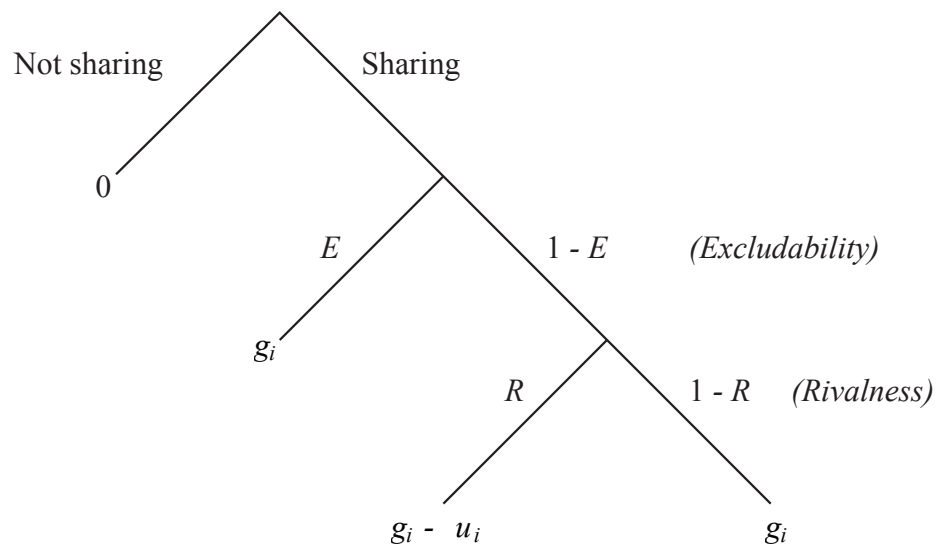

Figure 2: Sharing game tree

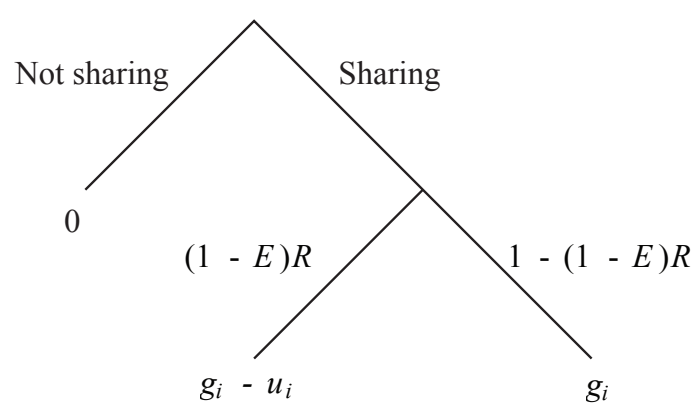

Figure 3: Simplified sharing game tree 
Proposition 1. In a non-repeated game without sharing constraint, a necessary condition for the consumer to share is that the reward of sharing is positive:

$$
g_{i} \geq 0
$$

Additionally, a consumer will share if and only if the reward of sharing exceeds the expected loss of utility caused by rivalness:

$$
g_{i} \geq(1-E) R u_{i}
$$

(Proof in the text above)

If the excludability is total $(E=1)$ the consumer is always willing to share, as long as $g_{i} \geq 0$. This is due to the fact that the producer is always able to monitor and exclude the illegitimate users of the digital good. Consequently, the consumer is always willing to share as long as it is not costly, since there is no risk that rivalness will take place ${ }^{11}$. The same situation occurs when there is no rivalness $(R=0)$.

If we leave aside the internal benefit/cost of sharing, $g_{i}$ - which depends on the preferences/position of each agent and not on the publicness - and only consider the external cost of sharing, $(1-E) R u_{i}$, we can notice that this external cost of sharing increases when the rivalness increases and decreases when excludability increases. The external cost also increases when the potential loss of utility, $u_{i}$ increases, thus the consumer is more likely to share if the utility brought by the digital good is low since, in this case, the potential loss in case of rivalness is also low.

As excludability and rivalness influence the external cost of sharing in opposite ways, it is interesting to investigate which of these effects is stronger. Starting from a point with no rivalness and excludability, if rivalness and excludability are increased at the same rate (i.e. $E \equiv R$ ), the increase in rivalness has a stronger effect on the external cost of sharing - which will thus increase - up to the point where $R=E=1 / 2$. After this point, if rivalness and excludability continue to increase at the same rate, excludability has a larger effect than rivalness and the cost of sharing diminishes up to the point where it is equal to zero when both $R$ and $E$ are equal to 1 . Thus if the firms are aiming at decreasing piracy by increasing the cost of sharing, it is not necessarily the case that they should simultaneously increase excludability and rivalness.

Likewise, if the firms are able to have complete control over who is using the good $(E=1)$, they could, by setting the rivalness equal to zero, use the consumers as a mean of distribution of the digital good. When $E=1$, the external cost of sharing is zero, and as long as $g_{i}$ is greater than zero, the consumers will be willing to let other consumers copy the good.

An opposite strategy also exists. Firms that fear that they will not be able to control closely enough who is using their product, could instead opt for a strategy with no excludability but a total rivalness. In this case, the firms would hand over the control of the digital good - but also the risks of loss due to piracy - to the consumers. As the digital good is completely rival, sharing consumers

\footnotetext{
${ }^{11}$ If a legitimate consumer lets another consumer copy the digital good, rivalness will not take place, even if rivalness techniques are used, since the producer will be able to detect the illegitimate user, and prevent her from using the good. In this case, the legitimate user remains the sole user of the good.
} 
have potentially a lot to loose and will certainly refuse to share unless they are compensated for the potential loss of utility - e.g. unless the other consumer buys the good from them. In this case, the digital good becomes quite similar to a private good and only consumers potentially suffer from piracy.

It is also worth noticing that this last type of strategy is likely to restrain the distribution of the digital good over the network and hence decrease the probability that a pirate consumer will find a source of digital good ${ }^{12}$. This phenomenon is particularly interesting when firms are able to have, at first, a high excludability level, but then tend to loose control over the good, resulting in a decrease of the level of excludability over the time.

\subsection{Pirating or not pirating?}

Assuming that the digital good is valuable for the consumer $\left(u_{i} \geq 0\right)$, that it is worth buying $\left(u_{i}-p \geq 0\right)$ and that it is actually costly to search for a pirate source $(s \geq 0)$, a consumer $i$ will never choose to pirate in two cases:

- If the consumer is not part of any network $\left(N_{i}=0\right)$.

- If none of the consumers is willing to share.

If the consumer is certain that all the other consumers will be willing to share, the net payoff for consumer $i$ when pirating is (c.f. Figures 4 and 5 ):

$$
N_{i}(1-E)(1-R) u_{i}-N_{i} c-s
$$

Where $N_{i}(1-E)(1-R) u_{i}$ is the expected utility gained when pirating and $N_{i} c-s$ is the expected cost of this activity. We can immediately notice that there is another case when the consumer will never pirate: when the expected utility of pirating is lower than the cost of pirating ${ }^{13}$ :

$$
N_{i}(1-E)(1-R) u_{i}<N_{i} c+s
$$

\subsubsection{Pirating in a perfectly connected network}

If the network is fully connected $\left(N_{i}=1, \forall i\right)$, the net payoff of pirating is:

$$
(1-E)(1-R) u_{i}-c-s
$$

Thus the consumer will pirate only if $(1-E)(1-R) u_{i} \geq c-s$. This definition of the pirating payoff gives some interesting insights about the piracy phenomenon. Indeed, this means that in the "best of the worlds" - e.g. the network is fully connected and other consumers are always willing to share the piracy decision is essentially based on the difference between the expected utility obtained when pirating and the utility obtained when buying, and on the

\footnotetext{
${ }^{12}$ If rivalness does not take place, consumers are more likely to share the good. As a consequence, a lot of consumers may be able to get a copy of the good. Even if we assume that they are excluded - and can not use the good - they can nevertheless share the good as well and become a source for other consumers. If at any point the excludability decreases, the piracy will be important, as the number of sources is high.

${ }^{13}$ We will assume in the following paragraphs that this is not the case, and that piracy is always a worthwhile option, e.g. the expected utility gained from pirating always exceeds the expected cost of pirating $\left(N_{i}(1-E)(1-R) u_{i} \geq N_{i} c+s\right)$.
} 
difference between the cost of legal purchasing and the cost of pirating. Indeed, the consumer decides to pirate if:

$$
(1-E)(1-R) u_{i}-c-s \geq u_{i}-p \Leftrightarrow(1-E)(1-R) u_{i}-u_{i} \geq c+s-p
$$

As $(1-E)(1-R) u_{i} \leq u_{i}$, the consumer will pirate if and only if $c+s \leq p$, i.e. if the cost of pirating is lower than the cost of purchasing the digital good legally. However, although $c+s \leq p$ is always a necessary condition for the consumer to pirate, it is a sufficient condition only when the publicness is total ( $R=0$ and $E=0)$. If there is no rivalness but excludability exists $(R=0$ and $E \in] 0,1])$, a sufficient condition for the consumer to pirate is: $c+s+E u_{i} \leq p$. Thus, pirating for piracy to occur the price of the digital good should be greater than the sum of the total cost of pirating and the expected loss of utility when the consumer pirates and is excluded. Intuitively, it means that if the excludability is very high, the firms will be able to charge a higher price without pushing consumers to pirate. On the contrary, if the excludability is very low, a price slightly above the cost of pirating will trigger a piracy behaviour.

The same kind of reasoning applies when both rivalness and excludability are present $(R \in] 0,1]$ and $E \in] 0,1])$. In this case, a sufficient condition for the consumer to pirate is: $c+s+(E+R-E R) u_{i} \leq p$. Thus the consumer will not pirate unless the official price is higher than the sum of the costs of pirating and the expected loss of utility when pirating and "losing" the good (due to exclusion or rivalness).

Let's assume that the firms choose as a price the highest price that does not cause the consumers to pirate:

$$
p=c+s+(E+R-E R) u_{i}-\epsilon \simeq c+s+(E+R-E R) u_{i}
$$

We can notice that the impact of increasing excludability (resp. rivalness) on the price depends on the level of rivalness (resp. excludability):

$$
\begin{aligned}
\frac{\partial p}{\partial E} & =(1-R) u_{i} \\
\frac{\partial p}{\partial R} & =(1-E) u_{i}
\end{aligned}
$$

Thus, increasing rivalness (resp. excludability) when excludability (resp. rivalness) is high will only have a small impact on the highest price that can be charged by the firms. Therefore it is probably more efficient for the firms, if we assume that increasing rivalness or excludability is costly, to adopt a "pure strategy" and to either increase excludability or rivalness, rather than both at the same time.

Formally, a sufficient condition for the consumer not to pirate is when either the rivalness or the excludability level is above an absolute critical value equal to $\frac{p-s-c}{u_{i}}$. If the level of rivalness (resp. excludability) reaches the critical value $R^{*}$ (resp. $\left.E^{*}\right)$, the consumer will never choose to pirate, regardless of the value of the level of excludability (resp. rivalness).

However, if neither of these levels reaches its absolute critical value, a second condition is required for the consumer not to pirate. For example, if the level of excludability is below the critical value $\left(E<\frac{p-s-c}{u_{i}}\right)$, then the level or rivalness 
would have to be at least equal to $R>\frac{p-s-c-E u_{i}}{(1-E) u_{i}}$ for the consumer not to pirate. Let's denote these two relative critical values $E^{*}(R)$ and $R^{*}(E)$.

Thus the sufficient conditions for the consumer not to pirate are:

- $R>R^{*}$ and $E \in[0,1]$

- $E>E^{*}$ and $R \in[0,1]$

- $R<R^{*}$ and $E>\frac{p-s-c-R u_{i}}{(1-R) u_{i}}$

- $E<E^{*}$ and $R>\frac{p-s-c-E u_{i}}{(1-E) u_{i}}$

With:

$$
\begin{aligned}
E^{*} & =\frac{p-s-c}{u_{i}} \\
R^{*} & =\frac{p-s-c}{u_{i}} \\
E^{*}(R) & =\frac{p-s-c-R u_{i}}{(1-R) u_{i}} \\
R^{*}(E) & =\frac{p-s-c-E u_{i}}{(1-E) u_{i}}
\end{aligned}
$$

It is interesting to note that the absolute critical values $E^{*}$ and $R^{*}$ decrease with $u_{i}, c$ and $s$ and increase with $p$. Thus from the firms' point of view, it is easier to deter piracy when the good is very valuable ( $u$ is high), or when the costs associated with piracy ( $s$ and $c$ ) are high. On the contrary, the higher the price of the digital good, $p$ is, the more difficult it will be to deter piracy, since the levels of rivalness or excludability will have to be higher in order to reach their critical value.

Likewise, if the first sufficient condition - i.e. $E>E^{*}$ or $R>R^{*}-$ is not met, the value of the minimum levels of the rivalness and excludability for the second set of sufficient conditions, $E^{*}(R)$ and $R^{*}(E)$, also depends negatively on $u, s$, and $c$. This means that the higher these three values are, the easier it is for the firms to prevent piracy since the level of excludability/rivalness required will be lower. On the contrary, the two critical values $E^{*}(R)$ and $R^{*}(E)$ depend positively on $p$, and thus when firms charge a higher price, higher levels of excludability or rivalness are required to prevent piracy. In addition, $E^{*}(R)$ and $R^{*}(E)$ depend negatively on each other $\left(\partial E^{*}(R) / \partial R<0\right.$ and $\left.\partial R^{*}(E) / \partial E<0\right)$ which means that a lower level of rivalness (resp. excludability) will be required to prevent piracy when the level of excludability (resp. rivalness) is high.

\subsubsection{Pirating in an imperfectly connected network}

Similar reasoning applies when the consumer is not in a fully connected network $\left(N_{i} \in[0,1[)\right.$. In this case the consumer will pirate if and only if (c.f. Figures 4 and 5):

$$
N_{i}(1-E)(1-R) u_{i}-N_{i} c-s \geq u_{i}-p
$$

In this case, a necessary condition for the consumer to pirate is:

$$
N_{i}(1-E)(1-R) u_{i}>N_{i} c+s
$$




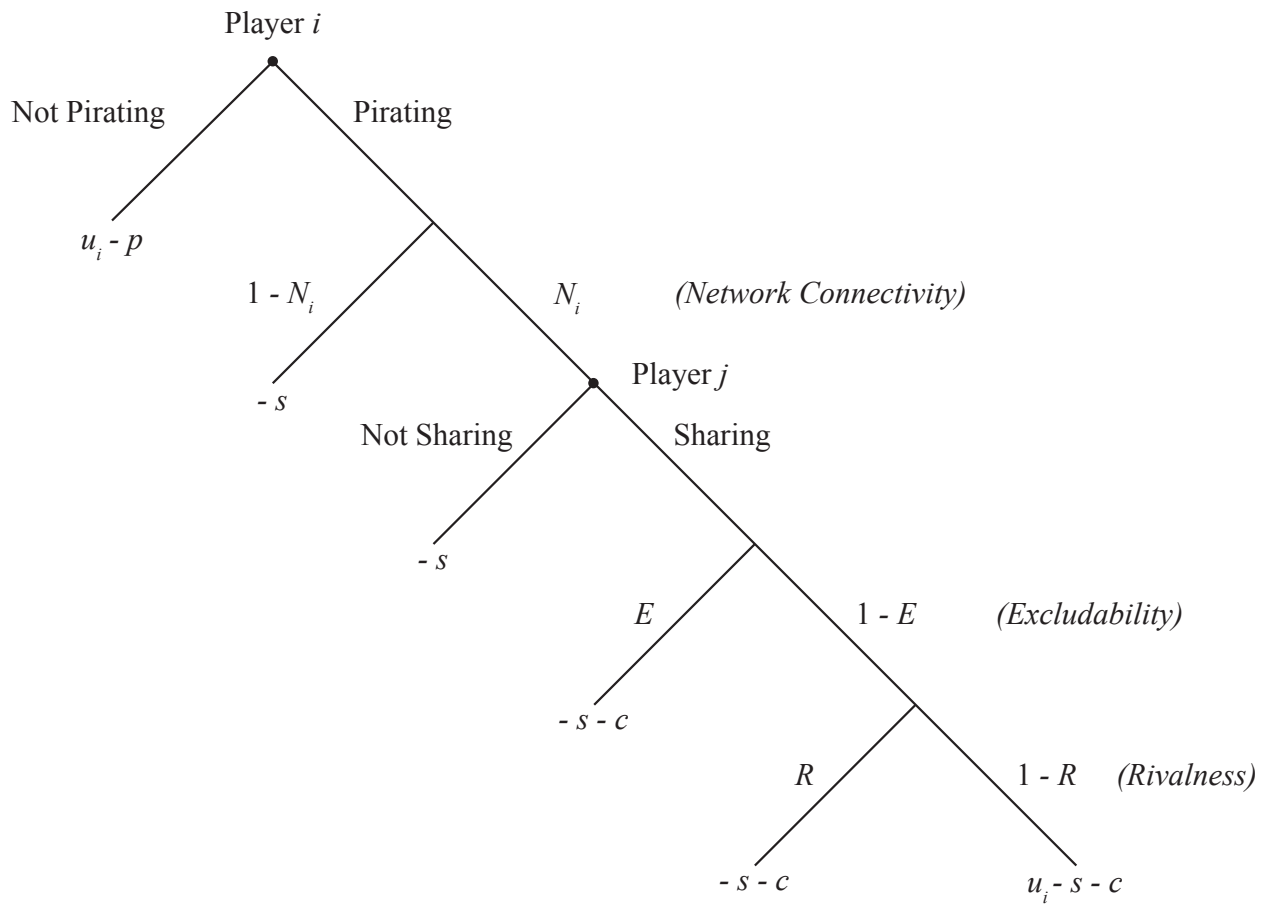

Figure 4: Pirating game tree

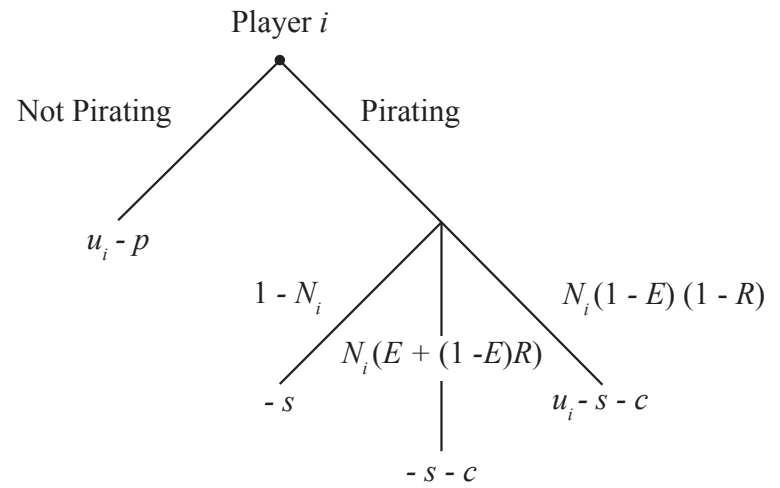

Figure 5: Simplified pirating game tree for player $i$ when player $j$ always shares. 
Which means, as we assumed before that the expected utility of pirating should be equal to or greater than the expected cost of pirating for the piracy behaviour to be worthwhile.

Therefore, a sufficient condition for the consumer to pirate is:

$$
p>\left(1-N_{i}(1-E)(1-R)\right) u_{i}+s+N_{i} c
$$

So if the official market price is higher than the expected cost - including the expected loss of utility - of piracy, then the consumer will decide to pirate. If we assume, as we did above, that the firms will charge a price just below the expected cost of pirating:

$p=\left(1-N_{i}(1-E)(1-R)\right) u_{i}+s+N_{i} c-\epsilon \simeq\left(1-N_{i}(1-E)(1-R)\right) u_{i}+s+N_{i} c$

We can notice that:

- Firms can charge a higher price when the good is more valuable for the consumers $\left(\partial p / \partial u_{i}>0\right)$.

- Firms can charge a higher price when the cost of pirating increases $(\partial p / \partial c>0$ and $\partial p / \partial s>0)$.

- Firms can charge a higher price when excludability and rivalness are high $(\partial p / \partial E>0$ and $\partial p / \partial R>0)$.

More interestingly, the impact of the connectivity of the network depends on the relative cost of copying and the expected utility of pirating:

$$
\frac{\partial p}{\partial N_{i}}>0 \Leftrightarrow c>(1-E)(1-R) u_{i}
$$

However, in this case, piracy would not be worthwhile since the cost of copying would outweigh the expected gain of utility. We can thus reasonably assume that $c<(1-E)(1-R) u_{i}$ and thus the impact of the connectivity of the network on the price is negative $\left(\partial p / \partial N_{i}<0\right)$. As a consequence, the less the network is connected, the higher the price charged by the firms can be.

In terms of network connectivity, it is possible to define a threshold level of network connectivity, $N_{i}^{*}$, below which, regardless of the level of publicness, the consumer will never pirate. This absolute critical value of network connectivity can be defined as:

$$
N_{i}^{*}=\frac{u_{i}-p+s}{u_{i}-c}
$$

Thus if $N_{i}<N_{i}^{*}$, the consumer will never pirate. However if the network is sufficiently connected $\left(N_{i} \geq N_{i}^{*}\right)$, the consumer may choose to pirate, but this decision also depends on the levels of excludability and rivalness. If $N_{i} \geq N_{i}^{*}$, a sufficient condition for the consumer not to pirate is that either the level of excludability or the level of rivalness reaches a relative critical value, $E^{*}\left(N_{i}\right)$ for the excludability, and $R^{*}\left(N_{i}\right)$ for the rivalness:

$$
E^{*}\left(N_{i}\right)=R^{*}\left(N_{i}\right)=\frac{p-s-N_{i} c-\left(1-N_{i}\right) u_{i}}{N_{i} u_{i}}
$$


Thus as long as $N_{i} \geq N_{i}^{*}$, a sufficient condition for the consumer not to pirate is that the level of rivalness (resp. excludability) is greater than $R^{*}\left(N_{i}\right)$ (resp. greater than $E^{*}\left(N_{i}\right)$ ). In this case, the level of excludability (resp. rivalness) can take any value between zero and one.

If this second condition is not met, it is possible to define a third sufficient condition based on the three levels (network, excludability and rivalness). If the network is sufficiently connected $\left(N_{i} \geq N_{i}^{*}\right)$ and rivalness and excludability are rather low $\left(E \leq E^{*}\left(N_{i}\right)\right.$ and $\left.R \leq R^{*}\left(N_{i}\right)\right)$ a sufficient condition for the consumer not to pirate is either:

$$
E>\frac{p-s-N_{i} c-\left(1-N_{i}(1-R)\right) u_{i}}{N_{i}(1-R) u_{i}}
$$

Or:

$$
R>\frac{p-s-N_{i} c-\left(1-N_{i}(1-E)\right) u_{i}}{N_{i}(1-E) u_{i}}
$$

This allows us to define a second set of relative critical values for the rivalness and excludability:

$$
\begin{aligned}
& E^{*}\left(N_{i}, R\right)=\frac{p-s-N_{i} c-\left(1-N_{i}(1-R)\right) u_{i}}{N_{i}(1-R) u_{i}} \\
& R^{*}\left(N_{i}, E\right)=\frac{p-s-N_{i} c-\left(1-N_{i}(1-E)\right) u_{i}}{N_{i}(1-E) u_{i}}
\end{aligned}
$$

It is also worth noticing that, regardless of the value of network connectivity, the consumer will also never pirate if one of the publicness level - either excludability or rivalness - is greater than the absolute critical value $E^{*}$ and $R^{*}$ defined in the Equations (1) and (2).

It means that in order for the consumer to adopt a piracy behaviour, the network should be sufficiently connected and the excludability and the rivalness should not be too high.

As all these critical levels depend on the value of the good for the consumers $(u)$, the price $(p)$, and the costs of pirating $(c$ and $s)$, it is possible to describe the influence of these factors on the critical values (Table 1 ).

Table 1: Impact of the variables on the absolute and relative critical values

\begin{tabular}{|c|c|c|c|c|}
\cline { 2 - 5 } \multicolumn{1}{c|}{} & $u_{i}$ & $p$ & $s$ & $c$ \\
\hline$N_{i}^{*}$ & + & - & + & + \\
\hline$E^{*}$ and $R^{*}$ & - & + & - & - \\
\hline$E^{*}\left(N_{i}\right)$ and $R^{*}\left(N_{i}\right)$ & - & + & - & - \\
\hline$E^{*}\left(N_{i}, R\right)$ & - & + & - & - \\
\hline$R^{*}\left(N_{i}, E\right)$ & - & + & - & - \\
\hline
\end{tabular}

Unsurprisingly, the presence of a more valuable good increases the critical value for the network connectivity, $N_{i}^{*}$, and decreases the critical values for excludability $\left(E^{*}, E^{*}\left(N_{i}\right), E^{*}(R)\right.$ and $\left.E^{*}\left(N_{i}, R\right)\right)$ and rivalness $\left(R^{*}, R^{*}\left(N_{i}\right)\right.$, $R^{*}(E)$ and $\left.R^{*}\left(N_{i}, E\right)\right)$. This means that if the good is very valuable to the 
consumer, the network connectivity has to be greater and the excludability and rivalness lower for the consumer to pirate. The same phenomenon occurs if the cost of pirating (either the search cost $s$ or the copying cost $c$ ) increases. In this case, the network connectivity also has to be higher - and rivalness and excludability lower - for the consumer to pirate. On the contrary, a higher price of the digital good will push the consumers to pirate even when the pirating conditions are "harsh", e.g. when the network connectivity is low and the rivalness and excludability are high.

Likewise, it is possible to analyse the impact that the level of connectivity of the network, the level of rivalness and the level of excludability have on each other (Table 2). It allows us to see that an increase in the network connectivity increases the relative critical values for excludability and rivalness $\left(E^{*}\left(N_{i}\right)\right.$, $E^{*}\left(N_{i}, R\right), R^{*}\left(N_{i}\right)$ and $\left.R^{*}\left(N_{i}, R\right)\right)$. This means that a consumer placed in a more connected network is more likely to pirate, even if the rivalness and excludability are high.

Table 2: Impact of the environmental variables on the relative critical values

\begin{tabular}{|c|c|c|c|}
\cline { 2 - 4 } \multicolumn{1}{c|}{} & $N$ & $E$ & $R$ \\
\hline$E^{*}\left(N_{i}\right)$ and $R^{*}\left(N_{i}\right)$ & + & & \\
\hline$R^{*}\left(N_{i}, E\right)$ & + & - & \\
\hline$E^{*}\left(N_{i}, R\right)$ & + & & - \\
\hline
\end{tabular}

The excludability and rivalness levels both have a negative effect (in terms of value) on each other's relative critical values. If the level of excludability increases, the relative critical values for rivalness $R^{*}(E)$ and $R^{*}\left(N_{i}, R\right)$ will decrease, which means that for the consumer to pirate when the level of excludability increases, the level of rivalness has to decrease. Likewise, an increase in rivalness will lead to a decrease in the relative critical values of excludability $E^{*}(R)$ and $E^{*}\left(N_{i}, R\right)$.

Proposition 2. In a non-repeated game without sharing constraint, any of the following conditions are sufficient conditions for the consumer not to pirate:

- The consumer $i$ is not part of a network $\left(N_{i}=0\right)$.

- The consumer $i$ is not connected to any consumer willing to share.

If the none of the above conditions are met, another sufficient condition for the consumer not to pirate is when either the network connectivity, excludability or rivalness reach an absolute critical value:

- The connectivity of the network, $N_{i}$, is below the absolute critical level $N_{i}^{*}\left(N_{i}<N_{i}^{*}\right)$ and the levels of excludability $(E)$ and rivalness $(R)$ are between zero and one.

- The level of excludability, $E$, is above the absolute critical level $E^{*}(E>$ $\left.E^{*}\right)$ and the level of network connectivity $\left(N_{i}\right)$ and the level of rivalness $(R)$ are between zero and one. 
- The level of rivalness, $R$, is above the absolute critical level $R^{*}\left(R>R^{*}\right)$ and the level of network connectivity $\left(N_{i}\right)$ and the level of excludability (E) are between zero and one.

These absolute critical values are defined by the equations (1), (2) and (5).

If the above conditions are not met and the network connectivity is rather high $\left(N_{i} \geq N_{i}^{*}\right)$, the following conditions are sufficient for the consumer not to pirate:

- The level of excludability, $E$, is above a relative critical level $E^{*}\left(N_{i}\right)$ (E> $E^{*}\left(N_{i}\right)$ ) and the level of rivalness $(R)$ is between zero and one.

- The level of rivalness, $R$, is above a relative critical level $R^{*}\left(N_{i}\right)(R>$ $R^{*}\left(N_{i}\right)$ ) and the level of excludibility $(E)$ is between zero and one.

- The level of excludability, $E$, is above a relative critical level $E^{*}\left(N_{i}, R\right)$ $\left(E>E^{*}\left(N_{i}, R\right)\right)$.

- The level of rivalness, $R$, is above a relative critical level $R^{*}\left(N_{i}, E\right)(R>$ $\left.R^{*}\left(N_{i}, E\right)\right)$.

These relative critical values are defined by the equations (6), (7) and (8). (Proof given in Appendix)

Corollary (1). The critical values $N_{i}^{*}, E^{*}\left(N_{i}\right), E^{*}\left(N_{i}, R\right), R^{*}\left(N_{i}\right), R^{*}\left(N_{i}, E\right)$, depend on the utility brought by the digital good $\left(u_{i}\right)$, on the cost of copying $(c)$, on the search cost ( $s)$, and on the price of the digital good ( $p)$ as follows:

- The absolute critical value for network connectivity, $N_{i}^{*}$, increases with the utility brought by the digital good $\left(u_{i}\right)$, and with the search cost $(s)$. This critical value decreases with the price of the digital good ( $p)$ and with the cost of copying (c).

- The critical values for excludability $\left(E^{*}, E^{*}\left(N_{i}\right)\right.$ and $\left.E^{*}\left(N_{i}, R\right)\right)$, and rivalness $\left(R^{*}, R^{*}\left(N_{i}\right)\right.$ and $\left.R^{*}\left(N_{i}, E\right)\right)$, decrease with the utility brought by the digital good $\left(u_{i}\right)$, with the cost of copying $(c)$, and with the search cost $(s)$. These critical values increase with the price of the digital good $(p)$.

Corollary (2). The relative critical values for excludability, $E^{*}\left(N_{i}\right)$ and $E^{*}$ $\left(N_{i}, R\right)$, and rivalness, $R^{*}\left(N_{i}\right)$ and $R^{*}\left(N_{i}, E\right)$, change depending on the environmental variables $N_{i}, E$ and $R$ as follows:

- The relative critical values for excludability, $E^{*}\left(N_{i}\right)$ and $E^{*}\left(N_{i}, R\right)$, increase with the level of network connectivity $N_{i}$ and decrease with the level of rivalness $R$.

- The relative critical values for rivalness, $R^{*}\left(N_{i}\right)$ and $R^{*}\left(N_{i}, E\right)$, increase with the level of network connectivity $N_{i}$ and decrease with the level of excludability E.

\section{Non-repeated game}

In this section we analyse the equilibria existing in a non-repeated game with two players. The following results can be generalised in a non-repeated $n$-player game. 


\subsection{Equilibria in the simple model}

The strategies of each player have two components based on whether they pirate $(P)$ or not $(\bar{P})$ and whether they share $(S)$ or not $(\bar{S})$. There are thus four different strategies available to each player: $\{P S, P \bar{S}, \bar{P} S, \bar{P} \bar{S}\}$. Table 3 shows the payoff matrix for two players.

Table 3: Payoffs Matrix

\begin{tabular}{|c|c|c|c|c|}
\cline { 2 - 5 } \multicolumn{1}{c|}{} & $P S$ & $P \bar{S}$ & $\bar{P} S$ & $\bar{P} \bar{S}$ \\
\hline$P S$ & $G_{P}+G_{S}, G_{P}+G_{S}$ & $-s+G_{S}, G_{P}$ & $G_{P}, G_{B}+G_{S}$ & $-s, G_{B}$ \\
\hline$P \bar{S}$ & $G_{P},-s+G_{S}$ & $-s,-s$ & $G_{P}, G_{B}+G_{S}$ & $-s, G_{B}$ \\
\hline $\bar{P} S$ & $G_{B}+G_{S}, G_{P}$ & $G_{B}+G_{S}, G_{P}$ & $G_{B}, G_{B}$ & $G_{B}, G_{B}$ \\
\hline $\bar{P} \bar{S}$ & $G_{B},-s$ & $G_{B},-s$ & $G_{B}, G_{B}$ & $G_{B}, G_{B}$ \\
\hline
\end{tabular}

With:

$G_{P}=N_{i}(1-E)(1-R) u_{i}-N_{i} c-s$

$G_{S}=g_{i}-(1-E) R u_{i}$

$G_{B}=u_{i}-p$

\subsubsection{Simultaneous game}

Assuming that these two players are playing once and simultaneously, we can notice that in the case where sharing is costly - e.g. when the expected payoff of sharing, $G_{S}$ is negative - all the strategies involving sharing ( $P S$ and $\bar{P} S$ ) are weakly dominated. In this case, and as long as the good is "worth the price" $\left(G_{B} \geq 0\right)^{14}$, the consumer will choose not to pirate. Thus when $G_{S}<0$ and $G_{B}>0,(\bar{P} \bar{S}, \bar{P} \bar{S})$ is a dominant strategy equilibrium.

However, as long as the expected payoff of pirating, $G_{P}$, is higher than the payoff of buying, $G_{B}$, this equilibrium is not Pareto optimal. Both consumers would be better off if they were both pirating and sharing $(P S, P S)$. However, this situation is not achievable due to the presence of a "temptation payoff": the payoff of both consumers is the highest when they pirate and do not share while the other one shares $\left(G_{P}>G_{P}+G_{S}\right)$. This is due to the fact that sharing is overall costly $\left(G_{S}<0\right)$. In this case, the players are in a prisoners' dilemma situation. They would both be better off cooperating (i.e. pirating and sharing), however they tend to free-ride on each other and end up in a sub-optimal situation.

If we assume, on the contrary, that there is a net benefit of sharing, $G_{S}>0$, the non-sharing strategies, $P \bar{S}$ and $\bar{P} \bar{S}$, are weakly dominated. In this case, the choice of strategy solely depends on the relative values of the pirating $\left(G_{P}\right)$ and buying $\left(G_{B}\right)$ payoffs:

- If $G_{P}>G_{B}$, both consumers will decide to pirate and $(P S, P S)$ is a (weakly) dominant strategy equilibrium.

- If $G_{P}<G_{B}$, both consumers will decide not to pirate and $(\bar{P} S, \bar{P} S)$ is a (weakly) dominant strategy equilibrium.

${ }^{14}$ When $G_{B} \geq 0, G_{B} \geq-s$ since by assumption $s \geq 0$. 
It is also possible to find Nash equilibria, in addition to the dominant strategy equilibria described above, in this game. The situation where none of the consumers pirate and none of the consumers share, $(\bar{P} \bar{S}, \bar{P} \bar{S})$, is always a Nash equilibrium. What is more, if there is a net benefit of sharing $\left(G_{S}>0\right)$, three additional Nash equilibria exist:

- $(\bar{P} S, P \bar{S})$ and $(P \bar{S}, \bar{P} S)$ : One of the consumers pirates and does not share whereas the other one does not pirate and shares.

- $(P S, P S)$ : provided that $G_{P}+G_{S}>G_{B}$, there is a Nash equilibrium where both consumers share and pirate.

Also, if the benefits of buying are higher than the benefits of pirating $\left(G_{P}<\right.$ $G_{B}$ ), then two other Nash equilibria exist:

- $(\bar{P} S, \bar{P} S)$ : since it is not worth pirating, nobody does. Since nobody pirates, everybody is willing to share even when sharing is costly $\left(G_{S}<0\right)$.

- $(\bar{P} S, \bar{P} \bar{S})$ and $(\bar{P} \bar{S}, \bar{P} S)$ : when nobody pirates, consumers are indifferent between sharing or not sharing. In this case, they either both share, both do not share, or one of them shares and the other does not.

All these results are summarised in the proposition below:

Proposition 3. When two consumers have the choice between pirating or not, and sharing or not, and when these choices are made only once and simultaneously, the following equilibria exist:

- When the expected payoff of sharing is negative $\left(G_{S}<0\right)$, there is only one equilibrium: $(\bar{P} \bar{S}, \bar{P} \bar{S})$ where both consumers do not pirate and do not share. This equilibrium is a dominant strategy equilibrium.

- When the expected payoff of sharing is positive $\left(G_{S} \geq 0\right)$, several equilibria exist as follows:

- $(\bar{P} \bar{S}, \bar{P} \bar{S})$ is a Nash equilibrium.

- $(P S, P S)$ is a (weakly) dominant strategy equilibrium when $G_{P}>$ $G_{B}$. In this case, both consumers pirate and share.

- $(\bar{P} S, \bar{P} S)$ is a (weakly) dominant strategy equilibrium when $G_{P}<$ $G_{B}$. In this case both consumers decide not to pirate and share. The following additional Nash equilibria also exist: $(\bar{P} S, \bar{P} S),(\bar{P} S, \bar{P} \bar{S})$ and $(\bar{P} \bar{S}, \bar{P} S)$. This is due to the fact that as nobody pirates, consumers are indifferent between sharing or not sharing. (Proof in the text above)

Corollary. When $G_{S}<0$ and $G_{P}+G_{S}>G_{B}$, the dominant strategy equilibrium $(\bar{P} \bar{S}, \bar{P} \bar{S})$ is not Pareto optimal. In this case, the Pareto optimal outcome is when both consumers pirate and share: $(P S, P S)$. However this outcome can never be achieved due to the fact that strategies involving sharing are always dominated. (Proof in the text above) 


\subsubsection{Sequential game}

Quite similar results are obtained when the game is played sequentially instead of simultaneously. When the benefits of piracy exceed the benefits of buying $\left(G_{P}>G_{B}\right)$ and sharing is costly $\left(G_{S}<0\right)$, the player placed in second position will never share, regardless of the decision of the first player. Also, the second player is always aware of whether the first player decided to share or not, and will decide to pirate only if the first player shares. The first player would prefer to pirate, but knows that, as sharing is costly, the second player will never decide to share ${ }^{15}$. As the game is not repeated, it is not possible for the first player to incite the second player to share, as even if the first player decides to share, not sharing is a dominant strategy for the second player. A simple backward induction reasoning shows that, in this situation, the first player will choose not to pirate and not to share $(\bar{P} \bar{S})$. As a consequence the second player will never choose to pirate, and - since the first player does not pirate - is indifferent between sharing or not. Thus when the payoff of pirating is higher than the payoff of buying, and when sharing is costly, there are two possible equilibria: $(\bar{P} \bar{S}, \bar{P} S)$ and $(\bar{P} \bar{S}, \bar{P} \bar{S})$.

If, on the contrary, sharing gives a positive payoff $\left(G_{S}>0\right)$, the second player will always choose to share, and will choose to pirate if she sees that the first consumer is sharing. Since sharing is beneficial, the first player will choose to share as well and, knowing that the second player is always sharing, will also decide to pirate. Consequently, as the first consumer shares, the second player will also decide to pirate. Thus when the payoff of pirating is higher than the payoff of buying, and when sharing is beneficial, the unique equilibrium is $(P S, P S)$.

If the benefits of piracy are lower than the benefits of buying $\left(G_{P}<G_{B}\right)$, both the first and second players will never choose to pirate. As such, regardless whether sharing is costly or not, they are indifferent between sharing and not sharing. Therefore in this case, there are four possible equilibria: $(\bar{P} \bar{S}, \bar{P} \bar{S})$, $(\bar{P} S, \bar{P} S),(\bar{P} S, \bar{P} \bar{S})$ and $(\bar{P} \bar{S}, \bar{P} S)$.

Proposition 4. When two consumers have the choice between pirating or not, and sharing or not, and when choices are made only once and sequentially, the following equilibria exist:

- $(\bar{P} \bar{S}, \bar{P} S)$ and $(\bar{P} \bar{S}, \bar{P} \bar{S})$, when $G_{P}>G_{B}$ and $G_{S}<0$.

- $(P S, P S)$, when $G_{P}>G_{B}$ and $G_{S}>0$.

- $(\bar{P} \bar{S}, \bar{P} \bar{S}),(\bar{P} S, \bar{P} S),(\bar{P} S, \bar{P} \bar{S})$ and $(\bar{P} \bar{S}, \bar{P} S)$, when $G_{P}<G_{B}$.

(Proof in the text above)

Corollary. When $G_{S}<0$ and $G_{P}+G_{S}>G_{B}$, the equilibria in the sequential game, $(\bar{P} \bar{S}, \bar{P} S)$ and $(\bar{P} \bar{S}, \bar{P} \bar{S})$ are not Pareto optimal. In this case, the Pareto optimal outcome is when both consumers pirate and share: $(P S, P S)$. (Proof in the text above)

\footnotetext{
${ }^{15}$ Unless the first player decides not to pirate, in which case the second player is indifferent between sharing and not sharing.
} 


\subsection{Impact of forced cooperation}

Following the corollary of Proposition 3 and 4, it is obvious that, from the consumers' point of view, one of the main problems with piracy is the free-riding occurring among consumers. As we can reasonably assume that in general the benefits of sharing for most of the people are, at best, null, and in most cases negative, this problem is very likely to arise. For this very reason, most of the pirate channels and peer-to-peer networks include mechanisms encouraging or even forcing cooperation among users. In this case, consumers that choose to pirate also necessarily have to share.

The game is thus simplified since the $P \bar{S}$ strategy does not exist anymore. If the expected payoff of sharing, $G_{S}$ is negative, the following equilibria exist:

- If $G_{B}>G_{P}+G_{S}$, e.g. the payoff of buying exceeds the total payoff of pirating and sharing, $(\bar{P} \bar{S}, \bar{P} \bar{S})$ is a dominant strategy equilibrium. In this case none of the consumers pirate nor share. The equilibrium is also the Pareto optimal outcome.

- If $G_{B}<G_{P}+G_{S}$, there is no dominant strategy equilibrium. However, $(\bar{P} \bar{S}, \bar{P} \bar{S})$ and $(P S, P S)$ are both Nash equilibria. The Pareto optimal outcome is $(P S, P S)$ and is therefore achievable.

If the expected payoff of sharing, $G_{S}$ is positive, the possible equilibria are:

- (PS, $P S)$ if $G_{P}>G_{B}$ : the consumers both pirate and share if the payoff of pirating is higher.

- $(\bar{P} S, \bar{P} S)$ if $G_{P}<G_{B}$ : the consumers both buy the good and share when the payoff of buying the good is higher.

All these results are summarised in Proposition 5.

Proposition 5. In a non-repeated simultaneous game, if a mechanism, forcing the consumers who decide to pirate to share, is introduced the following equilibria are obtained:

- $(\bar{P} \bar{S}, \bar{P} \bar{S})$ when $G_{S}<0$ and $G_{B}>G_{P}+G_{S}$.

- $(\bar{P} \bar{S}, \bar{P} \bar{S})$ and $(P S, P S)$ when $G_{S}<0$ and $G_{B}<G_{P}+G_{S}$.

- $(P S, P S)$ when $G_{S}>0$ and $G_{P}>G_{B}$.

- $(\bar{P} S, \bar{P} S)$ when $G_{S}>0$ and $G_{P}<G_{B}$.

(Proof in the text above)

Corollary. When $G_{S}<0$ and $G_{P}+G_{S}>G_{B}$, the Pareto optimal outcome $(P S, P S)$ can be achieved as $(P S, P S)$ is a Nash equilibrium. This is due to the fact that, as pirating consumers are forced to share, there is no possible free-riding among consumers.

There is little change in these results when the game is played sequentially instead of simultaneously. As in the case of the simultaneous game, forcing cooperation allows the consumer to achieve the Pareto optimal equilibrium $(P S, P S)$ when pirating is more advantageous $\left(G_{P}>G_{B}\right)$, sharing costly $\left(G_{S}<0\right)$, and 
pirating and sharing is overall more advantageous $\left(G_{P}+G_{S}>G_{B}\right)$. However, it is worth noticing that, on the contrary to the simultaneous game, a "cheating" option still exists for the second player, despite the forced cooperation, when the cost of sharing outweighs the benefit of pirating $\left(G_{P}+G_{S}<G_{B}\right)$. The second player can observe when the first player decides not to pirate, and in this case can himself choose to pirate without bearing the cost of sharing. For this reason, on the contrary to the simultaneous game with forced cooperation in which both players are usually indifferent between sharing or not in this case, the first player will necessarily choose not to share in order to avoid the loss caused by this behaviour of the second player.

Proposition 6. In a non-repeated sequential game, if a mechanism, forcing the consumers who decide to pirate to share, is introduced the following equilibria are obtained:

- $(\bar{P} \bar{S}, \bar{P} \bar{S}),(\bar{P} S, \bar{P} S),(\bar{P} S, \bar{P} \bar{S}),(\bar{P} \bar{S}, \bar{P} S)$ when $G_{B}>G_{P}$.

- $(\bar{P} \bar{S}, \bar{P} \bar{S})$ and $(\bar{P} \bar{S}, \bar{P} S)$ when $G_{B}<G_{P}$ and $G_{B}-G_{P}<G_{S}<0$.

- $(P S, P S)$ otherwise.

(Proof in the text above)

Corollary. When $G_{S}<0$ and $G_{P}+G_{S}>G_{B}$, the Pareto optimal outcome $(P S, P S)$ can be achieved since it is a Nash equilibrium.

As shown in Propositions 5 and 6, a pirate network with forced cooperation can lead to an equilibrium where both consumers pirate and share even when sharing is costly. This is typically the case when the total benefits of pirating and sharing outweigh the payoff of buying - e.g. when $G_{P}+G_{S}>G_{B}$.

For this condition to be true, the total cost of sharing $G_{S}=g_{i}-(1-E) R u_{i}$ should not be too high. Indeed, if $g_{i} \leq-p+s+c$, the intrinsic cost of sharing, $g_{i}$, is such that it exceeds the difference between the price of the good, $p$, and the total cost of pirating, $s+c$. In this case, the benefits of pirating will never outweigh the cost of sharing, even when the environmental conditions are the best for the consumer - i.e. when the network is fully connected $\left(N_{i}=1\right)$ and the ex-ante publicness is total $(E=0$ and $R=0)$.

If the intrinsic cost of sharing is rather low $\left(g_{i}>-p+c+s\right)$, another condition is required. The network needs to be sufficiently connected, as otherwise the consumer would pay the cost of sharing, but would not be rewarded as successful pirating would be quite unlikely. Thus if the connectivity of the network is below a critical value $N_{i}^{* *}$, the consumer will never choose to pirate and share as the benefits of pirating and sharing, $G_{P}+G_{S}$ are in this case smaller than the benefit of buying, $G_{B}$. This critical value $N_{i}^{* *}$ is defined as:

$$
N_{i}^{* *}=\frac{u_{i}-p+s-g_{i}}{u_{i}-c}
$$

$N_{i}<N_{i}^{* *}$ is a sufficient condition for the consumer not to pirate in the case of forced cooperation. However, if $N_{i} \geq N_{i}^{* *}$, other conditions are required. Indeed, when the network connectivity is rather large, the consumer will choose not to pirate only if the excludability and rivalness reach a sufficient level. As we did in the case without forced cooperation (Proposition 2), it is possible to 
define a relative critical level for the excludability, $E^{* *}\left(N_{i}\right)$, and the rivalness, $R^{* *}\left(N_{i}\right)$, such as if any of these two levels is reached, the consumer will never choose to pirate and share. These critical levels are defined as follows:

$$
E^{* *}\left(N_{i}\right)=R^{* *}\left(N_{i}\right)=\frac{p-s-N_{i} c-\left(1-N_{i}\right) u_{i}+g_{i}}{N_{i} u_{i}}
$$

Thus, $E>E^{* *}\left(N_{i}\right)$ is a sufficient condition for the consumer not to pirate. In this case, the level of excludability can take any value between zero and one. Likewise, $R>R^{* *}\left(N_{i}\right)$ is also a sufficient condition for the consumer not to pirate.

If this second condition is not reached - i.e. neither the level of excludability $E$ nor the level of rivalness $R$ reaches the critical value defined above - it is possible to define a third sufficient condition for the consumer not to pirate. As in this case the network connectivity is too high and both excludability and rivalness levels are too low, this third condition relies on two relative critical values for excludability and rivalness defined as follows:

$$
\begin{aligned}
& E^{* *}\left(N_{i}, R\right)=\frac{p-s-N_{i} c+g_{i}-\left(1-N_{i}(1-R)+R\right) u_{i}}{\left(N_{i}(1-R)-R\right) u_{i}} \\
& R^{* *}\left(N_{i}, E\right)=\frac{p-s-N_{i} c+g_{i}-\left(1-N_{i}(1-E)+E\right) u_{i}}{\left(N_{i}(1-E)-E\right) u_{i}}
\end{aligned}
$$

Thus, in the case of forced cooperation, $E>E^{* *}\left(N_{i}, R\right)$ or $R>R^{* *}\left(N_{i}, E\right)$ are sufficient conditions to deter consumers from pirating and sharing. These results are summarised in the proposition below.

Proposition 7. In a non-repeated simultaneous game with forced cooperation, a cooperative equilibrium, $(P S, P S)$, where both consumers pirate and share is obtained if and only if the total payoff of pirating and sharing, $G_{P}+G_{S}$, is greater than the payoff of buying, $G_{B}$. The following conditions are sufficient conditions for this former condition not to be true - i.e. are sufficient conditions for $G_{P}+G_{S}<G_{B}$ :

- $g_{i}<-p+s+c$ and $N_{i}, E, R \in[0,1]$

If this condition is not met, one of the following conditions is required:

- $N_{i}<N_{i}^{* *}$ and $E, R \in[0,1]$

- $N_{i} \geq N_{i}^{* *}$ and $E>E^{* *}\left(N_{i}\right)$ and $R \in[0,1]$

- $N_{i} \geq N_{i}^{* *}$ and $R>R^{* *}\left(N_{i}\right)$ and $E \in[0,1]$

- $N_{i} \geq N_{i}^{* *}$ and $E \leq E^{* *}\left(N_{i}\right)$ and $R>R^{* *}\left(N_{i}, E\right)$

- $N_{i} \geq N_{i}^{* *}$ and $R \leq R^{* *}\left(N_{i}\right)$ and $E>E^{* *}\left(N_{i}, R\right)$

The critical values $N_{i}^{* *}, E^{* *}\left(N_{i}\right), R^{* *}\left(N_{i}\right), E^{* *}\left(N_{i}, R\right)$ and $R^{* *}\left(N_{i}, E\right)$ are defined by the Equations (9), (10), (11) and (12).

Corollary (1). The critical values $N_{i}^{* *}, E^{* *}\left(N_{i}\right), R^{* *}\left(N_{i}\right), E^{* *}\left(N_{i}, R\right)$ and $R^{* *}\left(N_{i}, E\right)$ depend on the utility brought by the digital good $\left(u_{i}\right)$, on the cost of copying (c), on the search cost (s), on the price of the digital good (p), and on the net benefit/cost of sharing $\left(g_{i}\right)$ as follows: 
- The absolute critical value for network connectivity, $N_{i}^{* *}$, increases with the utility brought by the digital good $\left(u_{i}\right)$, with the search cost (s), and with the cost of copying (c). This critical value decreases with the price of the digital good $(p)$ and with the net benefit/cost of sharing $\left(g_{i}\right)$.

- The critical values for excludability, $E^{* *}\left(N_{i}\right)$ and $E^{* *}\left(N_{i}, R\right)$, and rivalness, $R^{* *}\left(N_{i}\right)$ and $R^{* *}\left(N_{i}, E\right)$, decrease with the utility brought by the digital good $\left(u_{i}\right)$, with the cost of copying (c), and with the search cost $(s)$. These critical values increase with the price of the digital good (p) and with the benefit of sharing $\left(g_{i}\right)$.

Corollary (2). The relative critical values for excludability, $E^{* *}\left(N_{i}\right)$ and $E^{* *}$ $\left(N_{i}, R\right)$, and rivalness, $R^{* *}\left(N_{i}\right)$ and $R^{* *}\left(N_{i}, E\right)$, change depending on the environmental variables $N_{i}, E$ and $R$ as follows:

- The relative critical values for excludability, $E^{* *}\left(N_{i}\right)$ and $E^{* *}\left(N_{i}, R\right)$, increase with the level of network connectivity $N_{i}$ and decrease with the level of rivalness $R$.

- The relative critical values for rivalness, $R^{* *}\left(N_{i}\right)$ and $R^{* *}\left(N_{i}, E\right)$, increase with the level of network connectivity $N_{i}$ and decrease with the level of excludability E.

It is useful to compare the critical value defined in Proposition 7, which defines the critical values of network connectivity, excludability and rivalness when forced cooperation exists, with the critical values without forced cooperation defined in Proposition 2. We find that, although forced cooperation allows to obtain a pirating-sharing equilibrium, it is also more sensitive to the environmental conditions. Indeed, as long as sharing is intrinsically costly $\left(g_{i}<0\right)$, the critical level of network connectivity below which the consumer will decide not to pirate is higher in the case of forced cooperation $\left(N_{i}^{* *}>N_{i}^{*}, \forall g_{i}<0\right)$, and the critical levels of excludability and rivalness above which the consumer will never pirate are both lower in the case of forced cooperation $\left(E^{* *}\left(N_{i}\right)>E^{*}\left(N_{i}\right)\right.$ and $\left.R^{* *}\left(N_{i}\right)>R^{*}\left(N_{i}\right), \forall g_{i}<0\right)$.

We can reasonably assume that for most of the consumers nowadays, the payoff of sharing is negative $\left(G_{S}<0\right)$ and the payoff of piracy usually outweighs the payoff of buying $\left(G_{P}>G_{B}\right)$. What is more, the cost of sharing is usually rather small, and we can assume that in most cases, the combined benefits of pirating and sharing are greater than the benefit of buying $\left(G_{P}+G_{S}>G_{B}\right)$. Nevertheless, the prospect of avoiding the cost of sharing certainly represents a serious temptation payoff for the individuals, especially once taken into account the fact that the cost of sharing includes the expected cost of some, unlikely but nevertheless potentially very high, criminal and civil charges. As most individuals are risk averse, they will try as much as possible to avoid sharing. In this case, regardless of the strategies of the firms, the pirate network would collapse. It is therefore very important for the creators of the network to insure that this does not happen by forcing the cooperation among users. As such, most of the peer-to-peer protocols nowadays include mechanisms preventing the users from downloading digital goods if they are not sharing. As shown in this section, such mechanisms prevent the free-riding behaviour among consumers and maximise the consumers' global welfare. 
However, in the real life, such mechanisms may not be sufficient. This is due to the fact that the speed at which consumers download (pirate) and upload (share) are often asymmetrical. It is thus possible that a consumer would finish downloading the sought digital good before she finishes to upload a single unit of the shared digital good. A consumer in this situation would be placed in a position similar to the second player in the sequential game without forced cooperation and will therefore immediately stop sharing. The other users will thus be harmed since they shared and did not obtain the pirated good ${ }^{16}$. This is a problem, since the most efficient users for the network - e.g. the ones with the highest sharing speed - are also the most likely to be harmed by such behaviour. In a small network, this problem can be solved by the repeated interactions among users.

\section{Repeated game}

We now assume that the game between the two players is repeated an infinite number of times $T(T=\infty) . \delta \in[0,1]$ is the discount factor used by the two players. We assume furthermore that sharing is intrinsically costly $\left(g_{i}<0\right)$ and that there is no forced cooperation mechanism.

The first, obvious, non-cooperative, equilibrium of such a game is the one that consists in playing the stage-game Nash equilibrium repeatedly:

Proposition 8. In an infinitely repeated game without forced cooperation and with a discount factor $\delta \in[0,1]$, the infinitely repeated stage-game Nash equilibrium - where both consumers do not pirate and do not share - is a subgame perfect Nash equilibrium (SPNE).

Corollary. The payoffs of the players at the stage-game Nash equilibrium are the minmax payoffs of the game. Each of these payoffs represents for each player the reservation utility as it is the worst outcome this player can be forced to take. Thus the repeated stage-game Nash equilibrium is the worst subgame perfect equilibrium (WSPE) (Fudenberg and Levine, 1983; Abreu, 1988) of the game and can be used as a punishment in order to incite cooperation. Grimtrigger, a.k.a. Nash reversion, based strategies use the payoffs of the WSPE as punishment payoffs.

\section{$5.1 \quad$ Sustainable piracy}

Using the previous corollary, we define a cooperative equilibrium, based on a simple grim-trigger punishment scheme, as follows:

- The player cooperates - e.g. pirates and shares - all the time as long as the other player cooperates - e.g. pirates and shares - as well.

- If the other player defects by pirating without sharing, the player subsequently stops pirating and sharing, and buys the good without sharing it in all subsequent periods.

\footnotetext{
${ }^{16} 99.99 \%$ of a software or a movie is, in general, as unusable as $0 \%$ of the same software or movie. This is actually the case with most of the binary files.
} 
Thus, in each subgame, the players have the choice between the following strategies $^{17}$ :

- Cooperating: The consumer pirates and shares in each subsequent period.

- Defecting: The consumer pirates without sharing. As a grim-trigger punishment is used by both players, the other player will then refuse to share in each subsequent period, thus the consumer will buy the good in each subsequent period.

- Buying: The consumer buys the good and does not share in this period or any subsequent one.

A cooperative strategy with grim-trigger punishment, $\sigma_{t}^{c}$, during the stage $t=k$ of the game can be defined as follows:

$$
\sigma_{t}^{c}= \begin{cases}P S & \text { If }(P S, P S) \text { was played at } t-1 \text { or if } t=1 \\ \bar{P} \bar{S} & \text { otherwise }\end{cases}
$$

Likewise, a defecting strategy at time $T=t, \sigma_{t}^{d}$, is defined as:

$$
\sigma_{t}^{d}= \begin{cases}P \bar{S} & \text { If }(P S, P S) \text { was played at } t-1 \text { or if } t=1 \\ \bar{P} \bar{S} & \text { otherwise }\end{cases}
$$

Finally, a "safe" strategy at time $T=t, \sigma_{t}^{s}-$ which consists in buying without sharing - is defined as:

$$
\sigma_{t}^{s}=\bar{P} \bar{S} \quad \forall t \in[1,+\infty[
$$

Assuming that the other player is cooperating and playing $\sigma_{t}^{c}$ at time $T=k$, the continuation payoff obtained when cooperating and playing $\sigma_{t}^{c}, \pi_{t}^{c}$, is defined as follows:

$$
\pi_{t}^{c}=\sum_{t=k}^{\infty} \delta^{t-k}\left(G_{P}+G_{S}\right)=\frac{G_{P}+G_{S}}{1-\delta}
$$

The continuation payoff of defecting and playing $\sigma_{t}^{d}$ at time $T=k, \pi_{t}^{d}$, is:

$$
\pi_{t}^{d}=G_{P}+\sum_{t=k+1}^{\infty} \delta^{t-k} G_{B}=G_{P}+\frac{\delta}{1-\delta} G_{B}
$$

These two payoffs need to be compared with the continuation payoff of the "safe" strategy, $\sigma_{t}^{s}$, at time $T=k, \pi_{t}^{s}$, which is the infinitely discounted sum of the stage-game minmax payoff $G_{B}$ :

$$
\pi_{t}^{s}=\sum_{t=k}^{\infty} \delta^{t-k} G_{B}=\frac{G_{B}}{1-\delta}
$$

Thus for a cooperating equilibrium with a grim-trigger punishment, $\left(\sigma_{t}^{c}, \sigma_{t}^{c}\right)$, to be sustainable at time $t=k$, the two following conditions need to be true:

1. $\pi_{t}^{c} \geq \pi_{t}^{d}$

\footnotetext{
${ }^{17}$ The other strategies are ignored as all of them are dominated
} 
2. $\pi_{t}^{c} \geq \pi_{t}^{b}$

Condition 2 simply requires that the combined payoff of pirating and sharing exceeds the payoff of buying:

$$
\pi_{t}^{c} \geq \pi_{t}^{s} \Leftrightarrow G_{P}+G_{S} \geq G_{B}
$$

Condition 1 is true if and only if:

$$
\pi_{t}^{c} \geq \pi_{t}^{d} \Leftrightarrow\left\{\begin{array}{c}
G_{P}+G_{S} \geq G_{B} \\
\text { and } \\
\delta \geq \frac{-G_{S}}{G_{B}-G_{P}}
\end{array}\right.
$$

Thus, in fact only two conditions are required for a cooperative equilibrium with grim-trigger punishment to be sustainable:

1. $G_{P}+G_{S} \geq G_{B}$

2. $\delta \geq \frac{-G_{S}}{G_{B}-G_{P}}$

The requirements for the first condition, $G_{P}+G_{S} \geq G_{B}$, to be true were discussed in detail in Proposition 7. However, it would be interesting to know the impact of the variables on the second condition. Based on this second condition, we define a critical value for the discount factor, $\delta^{*}$, above which the cooperative equilibrium will be sustainable:

$$
\delta^{*}=\frac{-G_{S}}{G_{B}-G_{P}}
$$

In regards to the environmental variables, we find that $\delta^{*}$ increases with the excludability $E$ and rivalness $R$, and thus decreases with the level of publicness. This makes sense as a decrease in publicness means more risk for the pirating and sharing consumers. Thus a higher discount ratio is required for them to commit to a cooperating equilibrium where they both pirate and share. An increase of network connectivity $N$ leads, on the contrary to a decrease of the critical discount ratio $\delta^{*}$. This result is not surprising since more network connectivity increases the chance for piracy to be successful and thus increases the expected utility of piracy-based strategies. As such, a lower discount ratio is required when more network connectivity is present in order for a pirating and sharing strategy to be sustainable.

The critical discount ratio $\delta^{*}$ is also increasing when the value of the good $u_{i}$ increases. As the potential loss due to exclusion or rivalness becomes higher, this higher loss needs to be compensated by a longer expected cooperation between the players. Likewise, an increase of the search costs, $s$, and the copy cost, $c$, also tend to increase $\delta^{*}$. This is logical since these two variables are part of the cost of pirating. Thus, when they increase, they make the cooperating strategy - which involves sharing and pirating - less interesting for the consumers unless the fruits of this strategy are collected during more periods. Contrariwise, both an increase in the official price of the good, $p$, and in the intrinsic benefit/cost of sharing will lead to a decrease of $\delta^{*}$. Quite trivially, the more the price of the digital good increases, the more pirating is an interesting option for the consumers. Thus, the more the price increases, the shorter the interaction between players can be, since even a few interactions will make worthwhile cooperating 
and pirating in order to avoid paying the very high official price. An increase in $g_{i}{ }^{18}$ also decreases the requirement in terms of duration of cooperation as it decreases the cost of sharing, thereby making the cooperating strategy more interesting even when cooperation does not last. These results are summarised in the proposition below:

Proposition 9. In an infinitely repeated game without forced cooperation and with a discount factor $\delta \in[0,1]$, the strongly symetric cooperative equilibrium $\left(\sigma^{c}, \sigma^{c}\right)$ - where consumers both pirate and share - based on a cooperative strategy profile with grim-trigger punishment $\sigma^{c}$ is sustainable if and only if the two following conditions are met:

- The combined payoff of pirating and sharing once is higher than the payoff of buying once: $G_{P}+G_{S}>G_{B}$.

- The discount ratio $\delta$ is higher than a critical value $\delta^{*}: \delta \geq \delta^{*}$

When these two conditions are met, the cooperative equilibrium $\left(\sigma^{c}, \sigma^{c}\right)$ is a subgame perfect Nash equilibrium (SPNE) of the infinitely repeated game.

The cooperative strategy with grim-trigger punishment $\sigma^{c}$ is defined by the Equation (13) and the critical value $\delta^{*}$ is defined by the Equation (19). (Proof in the text above)

Corollary (1). The critical value of the discount factor $\delta^{*}$ depends on the utility brought by the digital good $\left(u_{i}\right)$, the cost of copying $(c)$, the search cost $(s)$, the price of the digital good $(p)$ and the benefit/cost of sharing $\left(g_{i}\right)$ as follows:

- $\delta^{*}$ increases with the utility $u_{i}$, the cost of copying $c$, and the search cost $s$.

- $\delta^{*}$ decreases with the price $p$ and the benefit/cost of sharing $g_{i}$.

(Proof given in Appendix)

Corollary (2). The critical value of the discount factor $\delta^{*}$ change depending on the environmental variables $N_{i}, E$ and $R$ as follows:

- $\delta^{*}$ increases with the excludability $E$ and the rivalness $R$.

- $\delta^{*}$ decreases with the network connectivity $N_{i}$.

(Proof given in Appendix)

Proposition 9 shows that it is possible, even when sharing is costly, to obtain a cooperative equilibrium, where consumers pirate and share at the same time, as long as the benefits of pirating and sharing outweigh the benefits of buying, and as long as the discount ratio - which represents the probability for the game to be repeated or the patience ${ }^{19}$ of the consumers - exceeds a critical level $\delta^{*}$.

\footnotetext{
${ }^{18} g_{i}$ represents the intrinsic benefit or cost of sharing. It is a cost when $g_{i}<0$. In this case, when $g_{i}$ increases, it means that the cost of sharing is decreasing.

${ }^{19}$ When $\sigma$ is close to one, the consumer is infinitely patient, since all the gains in a future are worth as much that the gains in the present. On the contrary, is $\sigma$ is close to zero, it means that the consumers is highly impatient, since all the gains in the future are worth nothing and only the gains in the present count.
} 


\subsection{Sustainable piracies}

The cooperative equilibrium described by Proposition 9 is the simplest cooperative equilibrium (consumers pirate and share all the time) triggered by the simplest form of punishment (in case of defection consumers punish each other forever by not cooperating) of the repeated games. Other types of subgame perfect Nash equlibrium, involving more complex cooperative equilibrium behaviours - e.g. consumers mix strategies and are not always pirating and sharing - and/or more complex punishments - for example, the cheating consumer may be forgiven after some time - may also exist. Last but not least, the simple cooperative equilibrium of Proposition 9 is a symmetric equilibrium in the sense that the strategies of the players are identical and, at the equilibrium, their payoffs are identical. This section will show that sustainable non-symetrical subgame perfect Nash equilibria also exit.

Proposition 9 shows that a cooperative equilibrium with constant cooperation can be a SPNE. However cooperating all the time is not a necessary condition in order to get a SPNE. The well-known Folk Theorem (Friedman (1971), Fudenberg and Maskin (1986), Rubinstein (1979)) shows that as long as the discount factor is close to one - e.g. as long as the players are patient enough - many SPNE exist. In fact all pairs of strategies leading to an average discounted payoff greater than the repeated stage-game Nash equilibria minmax payoff can be SPNE when the discount ratio is high. The proposition below details this result:

Proposition 10 (The Folk Theorem). Let $\nu_{i}^{\infty} \equiv \nu_{i}^{0}, \nu_{i}^{1}, \ldots$ be an infinite stream of feasible and individually rational stage-game payoffs for player $i . \hat{\nu}_{i}^{\infty}$ is the average discounted payoff of this infinite stream of payoffs and is defined as follows:

$$
\hat{\nu}_{i}^{\infty}=(1-\delta) \sum_{t=0}^{\infty} \delta^{t} \nu_{i}^{t}
$$

If $\hat{\nu}_{i}^{\infty}$ is greater for all players $i$ than the stage payoff of the infinitely repeated stage-game Nash equilibrium $\left(\sigma^{s}, \sigma^{s}\right)-$ e.g. if:

$$
\hat{\nu}_{i}^{\infty}>G_{B}, \forall i
$$

and if the combined payoff of pirating and sharing, $G_{P}+G_{S}$, is higher than the payoff of buying, $G_{B}$, there exists a $\underline{\delta}<1$ such that, for all $\delta>\underline{\delta},\left(\hat{\nu}_{1}, \hat{\nu}_{2}\right)$ is the pair of average payoffs for players 1 and 2 arising in an SPNE. (Proof given in Appendix).

Corollary (1). As long as the combined payoff of pirating and sharing, $G_{P}+G_{S}$ is higher than the payoff of buying, $G_{B}$, and provided that the discount factor $\delta$ is sufficiently close to 1 , any pair of strategies $(\hat{\sigma}, \hat{\sigma})$ containing at least one episode of cooperative piracy (when both consumers pirate and share during the same period) is a SPNE. (Proof given in Appendix).

Corollary (2). As long as the payoff of pirating and sharing, $G_{P}+G_{S}$ is higher than the payoff of buying, $G_{B}$, and provided that the discount factor $\delta$ is sufficiently close to 1 , any pair of strategies $(\hat{\sigma}, \hat{\sigma})$ based on infinitely repeated sequences of actions containing at least one planned episode of reciprocal cooperative defection (when both players defect once in turn) is a SPNE. (Proof given in Appendix). 
Corollary (1) of Proposition 10, is a very important result since it shows that any pair of strategies containing at least one episode of cooperative piracy is sustainable as long as the discount ratio is high. This means that if players are expecting cooperation to last long, there is no need for them to pirate all the time for a cooperative equilibrium to be sustainable: they can agree to pirate and share only from time to time. This is particularly relevant in nowadays situation where firms, government or internet service providers (ISP) are monitoring the networks in order to find and to take pirating consumers to court. Consumers can devise strategies which are more difficult to detect than if they were pirating and sharing all the time. This also shows that the "punishment" strategies designed by some governments and ISP consisting in temporarily interrupting the internet access of pirating consumers - or threatening to do so - may not be a sufficient deterrent since consumers can still buy the good during a few periods and then resume pirating: preventing one way or another the consumers from pirating during a period of time (even when this period lasts for a long time) does not prevent the consumers from establishing cooperative pirating strategies since all the strategies containing at least one episode of cooperative piracy are sustainable.

Last but not least, this first Corollary is very important because it shows that there is not a unique piracy behaviour consisting in pirating and sharing all the time, but instead an whole range of pirating behaviours exist, from the very casual one to the most intensive one. This explains why, in industrialised countries, nearly all consumers pirated at least once in their life, and why some of them pirate very rarely while others pirate all the time. The problem for the firms supplying digital goods is that the behaviours - e.g. the frequency and the occurrence of cooperative piracy episodes - as well as their distribution in the consumers' population, is essentially unpredictable. This obviously makes it even more difficult to devise anti-piracy strategies.

Corollary (2) goes a step further in the direction of unpredictability, since it shows that the consumers do not even need to pirate at the same time for a cooperative equilibrium to be sustainable. It is indeed possible for the consumers to plan some episodes of reciprocal cooperative defection, when consumers alternatively defect in turn - e.g. one consumer is pirating without sharing, and the other one is sharing and buying, and then the roles are reversed - and include these episodes in their strategies. Corollary (2) shows that when at least one of these episodes is included in the strategies, they lead to a sustainable cooperative equilibrium. This result is particularly relevant in the - quite realistic - case when firms, governments or ISP are not able to monitor all the consumers at the same time, and/or are not able to monitor all the activities of the consumers at the same time (e.g. they can detect sharing but not pirating, for example), since consumers can adapt their piracy behaviour in function of the monitoring. What is more, it is quite common that, depending on the country considered, only one type of activities - e.g. pirating or sharing - is illegal. In all cases, this result shows that the consumers have even more options available in the way they design their pirating strategies. It is also worthwhile noticing that the two defections of the planned episode of reciprocal cooperative defection do not even need to be consecutive, which makes the whole set of possible strategies even less predictable by the firms or governments.

In addition, Corollaries (1) and (2) only consider sustainable equilibria with symmetrical payoffs. However, Proposition 10 shows that the payoffs of the 
players do not have to be identical: as long as each player earns on average at least as much as the WSPE stage-game payoff, the equilibrium is enforceable. Figure 6 shows the set of all the payoffs which are supportable as SNPE average payoffs when $\delta$ is close to one.

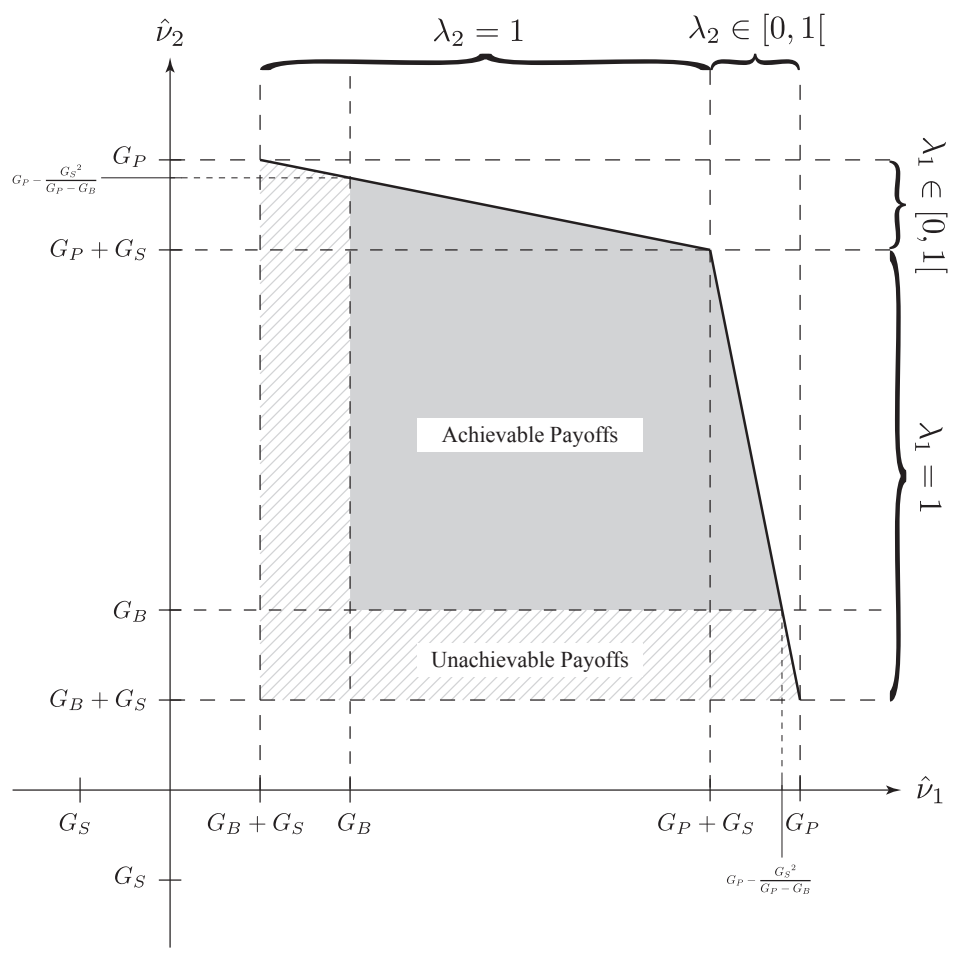

Figure 6: The Folk Theorem - set of supportable SPNE average payoffs when $\delta \rightarrow 1$

In order to find the set of supportable cooperative payoffs - and more particularly the set of maximum supportable cooperative payoffs - we assume that the two players are mixing the two strategies $^{20}$ :

- $P S$ : pirate and share

- $\bar{P} S$ : buy and share

The strategy $P S$ is played by the player $i$ with probability $\lambda_{i}$ and the strategy $\bar{P} S$ with probability $1-\lambda_{i}$. The more player $i$ shares without pirating - e.g. when $\lambda_{i} \rightarrow 0$ - the higher the average discounted payoff of player $j$ is, since she can pirate without having to bear the cost of sharing. However, one should notice that the player $i$ will never choose a value of $\lambda_{i}$ that would lead to an average discounted payoff lower than the minmax payoff $G_{B}$. By minimising $\lambda_{i}$ for a $\lambda_{j}=1$ and under the constraint that the average discounted payoff

${ }^{20}$ Mixing the two strategies $\bar{P} S$ and $P S$ is equivalent to mixing the three strategies $P \bar{S}, \bar{P} S$ and $P S . \bar{P} \bar{S}$ is used in both cases as a punishment. 
of player $i$ should be at least equal to the minmax payoff $G_{B}$, it is possible to define the maximum supportable SNPE average discounted payoff for player $j$.

Proposition 11 (Maximum supportable SPNE average payoff). The maximum supportable SPNE average payoff of the infinitely repeated game when the discount factor $\delta$ is close enough to one is:

$$
\hat{\nu}_{i}^{\max }=G_{P}-\frac{G_{S}{ }^{2}}{G_{P}-G_{B}}
$$

This maximum supportable SPNE average payoff is achieved for player $i$ when both players $i=1,2$ play $P S$ with probability $\lambda_{i}$ and $\bar{P} S$ with probability $\left(1-\lambda_{i}\right)$ and:

$$
\left\{\begin{array}{l}
\lambda_{i}=1 \\
\lambda_{j}=\lambda^{*}
\end{array}\right.
$$

With:

$$
\lambda^{*}=\frac{-G_{S}}{G_{P}-G_{S}}
$$

(Proof given in Appendix).

Corollary. When the difference between the payoff of pirating and the payoff of buying is large, or when cost of sharing is sufficiently small, the payoff of pirating, $G_{P}$, is the Maximum supportable SPNE average payoff:

$$
\hat{\nu}_{i}^{\text {max }} \simeq G_{P} \text { when }\left\{\begin{array}{cl}
\left(G_{P}-G_{B}\right) & \rightarrow \infty \\
\text { or } & \\
G_{S} & \rightarrow 0
\end{array}\right.
$$

(Proof given in Appendix).

Proposition 11 shows that the maximum supportable average discounted payoff for player $i, \nu_{i}^{\max }$, depends negatively on the cost of sharing, $G_{S}$, and positively on the difference between the gains of piracy and the gains of buying, $G_{P}-G_{S}$. The corollary shows that when the cost of sharing is proportionally very low, or when the difference between the payoff of pirating and the payoff of sharing is proportionally very large, this maximum supportable average payoff gets close to $G_{P}$ which is the highest possible payoff. Thus when the consumers are patient enough, and when they feel that pirating is much more advantageous than buying - or if the total cost of sharing is null - an equilibrium for which one of the players gets on average the pirating payoff is sustainable.In this equilibrium, one of the players always shares and virtually never pirates. As such, the payoff of the other player is on average equal to the pirating payoff. In this case, the full range of possible sustainable payoffs ${ }^{21}$ and possible sustainable strategies is available.

As for the non-repeated version of the game, it is important to determine which of the payoffs are Pareto optimal. The Proposition 12 below shows that the payoffs situated on the outer boundary of the set of possible average payoffs shown in Figure 6 are Pareto optimal payoffs. Any possible pair of payoffs situated inside the set is Pareto dominated by at least one other pair of payoffs situated on the outer boundary. Quite trivially, any inner pair of payoff can

\footnotetext{
${ }^{21}$ In this case the "unachievable payoffs" zone shown on Figure 6 becomes very small.
} 
not be Pareto optimal as it is possible to increase the payoff of player $i$ by increasing $\lambda_{i}$ without decreasing the payoff of player $j$, since $\lambda_{j}$ can be increased to compensate the raise in $\lambda_{i}$. On the contrary, for any pair of payoff situated on the outer boundary, it is not possible to compensate an increase of $\lambda_{i}$ (resp. $\lambda_{j}$ ) by an increase of $\lambda_{j}$ (resp. $\lambda_{i}$ ) since, by definition, a pair of payoff is situated on the boundary if and only if $\lambda_{j}$ (resp. $\lambda_{i}$ ) is equal to one. Therefore, for any pair of payoff situated on the outer boundary, it is not possible to increase the payoff of one of the consumers without decreasing the payoff of the other one. Payoffs on the outer boundary are thus Pareto optimal payoffs.

Proposition 12 (Pareto Optimality). When the discount factor $\delta$ is close enough to one, and the players $i=1,2$ play the following cooperative mixed strategy $\sigma_{i}$ :

- Play PS with probability $\lambda_{i}$

- Play $\bar{P} S$ with probability $\left(1-\lambda_{i}\right)$

The average discounted payoffs $\left(\hat{\nu}_{i}, \hat{\nu}_{j}\right)$ brought by the pair of strategies $\left(\sigma_{i}, \sigma_{j}\right)$ such as $\lambda_{i}=1$ and $\lambda_{j} \in[0,1]$ or $\lambda_{i} \in[0,1]$ and $\lambda_{j}=1$ are the Pareto optimal payoffs of the game.

(Proof given in Appendix)

Corollary. A pair of average discounted payoffs $\left(\hat{\nu}_{i}, \hat{\nu}_{j}\right)$ is a pair of Pareto optimal payoffs if and only if:

$$
\begin{cases}\hat{\nu}_{i}=\frac{G_{S}}{G_{P}-G_{B}} \hat{\nu}_{j}+\frac{\left(G_{P}-G_{B}-G_{S}\right)\left(G_{P}+G_{S}\right)}{G_{P}-G_{B}} & \forall \hat{\nu}_{j} \in\left[G_{B}+G_{S}, G_{P}+G_{S}\right] \\ \hat{\nu}_{i}=\frac{G_{P}-G_{B}}{G_{S}} \hat{\nu}_{j}+\frac{\left(G_{P}-G_{B}-G_{S}\right)\left(G_{P}+G_{S}\right)}{-G_{S}} & \forall \hat{\nu}_{j} \in\left[G_{P}+G_{S}, G_{P}\right]\end{cases}
$$

(Proof given in Appendix)

However, not all the Pareto optimal payoffs of the game are supportable as average subgame perfect Nash equilibria. The results of Proposition 10 and 12 are used to define the set of Pareto optimal SPNE of the infinitely repeated game. As achievable payoffs should be based on mixed strategies such as $\lambda_{i} \geq$ $\lambda^{*}, i=1,2$, this necessary condition is used in Proposition 13 to identify Pareto optimal payoffs that are supportable as equilibria, and thus the SPNE that are Pareto optimal.

Proposition 13 (Pareto Optimal SPNE). When the discount factor $\delta$ is close enough to one, and the players $i=1,2$ play $P S$ with probability $\lambda_{i}$ and $\bar{P} S$ with probability $\left(1-\lambda_{i}\right)$, the Pareto optimal payoffs brought by the pair of strategies $\left(\sigma_{i}^{*}, \sigma_{j}^{*}\right)$ such as $\lambda_{i}=1$ and $\lambda_{j} \in\left[\lambda^{*}, 1\right]$ or $\lambda_{i} \in\left[\lambda^{*}, 1\right]$ and $\lambda_{j}=1$ are supportable as average discounted subgame perfect Nash equilibrium payoffs. Thus all the subgame Nash equilibria $\left(\sigma_{i}^{*}, \sigma_{j}^{*}\right)$ are Pareto optimal equilibria.

(Proof given in Appendix).

Corollary (1). A subgame perfect Nash equilibrium based on the strategy profiles $\left(\sigma_{i}^{*}, \sigma_{j}^{*}\right)$ is Pareto optimal if an only if the equilibrium average discounted payoffs $\left(\hat{\nu}_{i}^{*}, \hat{\nu}_{j}\right)^{*}$ are such as:

$$
\left\{\begin{array}{l}
\hat{\nu}_{i}^{*}=\frac{G_{S}}{G_{P}-G_{B}} \hat{\nu}_{j}^{*}+\frac{\left(G_{P}-G_{B}-G_{S}\right)\left(G_{P}+G_{S}\right)}{G_{P}-G_{B}} \forall \hat{\nu}_{j}^{*} \in\left[G_{B}, G_{P}+G_{S}\right] \\
\hat{\nu}_{i}^{*}=\frac{G_{P}-G_{B}}{G_{S}} \hat{\nu}_{j}^{*}+\frac{\left(G_{P}-G_{B}-G_{S}\right)\left(G_{P}+G_{S}\right)}{-G_{S}} \forall \hat{\nu}_{j}^{*} \in\left[G_{P}+G_{S}, G_{P}-\frac{G_{S}{ }^{2}}{G_{P}-G_{B}}\right]
\end{array}\right.
$$




\section{(Proof given in Appendix)}

Corollary (2) (Pareto Perfection and Renegotiation-Proofness). The Pareto optimal subgame perfect Nash equilibria defined in Proposition 13 are renegotiation-proof. As such, they are Pareto perfect equilibria.

(Proof given in Appendix)

Proposition 13 shows that, when the consumers are patient enough, an extensive and varied range of Pareto optimal payoffs can be obtained as average payoffs of a subgame perfect Nash equilibrium. What is more, these Pareto optimal outcomes do not require to be symmetric. Indeed, Corollary (2) shows that any of the Pareto subgame perfect Nash equilibria are renegotiation-proof. Thus, these outcomes are stable even if they involve quite unequal equilibrium average payoffs. Hence, a situation where one of the players pirates all the time, while the other one shares all the time and buys and nearly all the time (and thus nearly never pirates) is an achievable, stable, and optimal cooperative equilibrium.

As a summary, this section shows that as long as consumers are patient and/or are expecting the game to last for a long time, an large number of cooperative pirating strategies exist. In addition to the nearly infinite number of sequences of cooperative piracy that can be designed, a very large number of Pareto optimal payoffs can be supportable as equilibria. All this shows that there is certainly not only one piracy behaviour that the firms producing digital goods should be afraid of, but instead that many different piracy behaviours are likely to exist. As such, monitoring, detecting and forecasting piracy behaviours is certainly unachievable.

\section{Analysis}

\subsection{Impact of the anti-piracy strategies}

If we assume that the consumers' pirating activity is based on simple nonrepeated interactions, as described in Sections 4.1.1 and 4.1.2, it should be relatively easy for the firms to prevent piracy. Indeed, they only have to ensure that the payoff of sharing, $G_{S}$ is negative. In this case, as shown in Propositions 3 and 4 , even when the combined benefits of pirating and sharing are higher than the benefits of buying the digital good $\left(G_{P}+G_{S}>G_{B}\right)$, the consumers are placed in a prisoner's dilemma situation and will refuse to share.

The conditions required for $G_{S}$ to be negative are described in section 3.1. If we assume that sharing is intrinsically costly for the consumers - i.e. $g_{i}<0$ - the firms actually do not have to do anything in order to prevent piracy. Indeed, in this case, the payoff of pirating $G_{S}$ is negative even when the levels of excludability and rivalness are null - i.e. when the ex-ante level of publicness is total. In this situation, the ex-post level of publicness is null since none of the consumers are sharing. As a logical consequence, none of the consumers would be pirating.

On the contrary, if consumers get a direct reward from sharing - i.e. $g_{i}>0$ - the payoff of sharing, $G_{S}$ is positive when the rivalness and excludability are null. Thus, when the ex-ante publicness is total and the intrinsic gain of sharing 
is positive, the ex-post publicness - for a sufficiently connected network - is also total. As shown in Proposition 3, consumers in this situation are likely to both share and pirate. In this case, the firms need to adopt anti-piracy strategies so that consumers stop pirating and/or sharing.

In order to prevent consumers from sharing, firms should decrease the $e x$ ante publicness by increasing the level of rivalness. We can reasonably assume that for most of the consumers, and in most cases, the intrinsic reward brought by the sharing activity is likely to be small, and as such, a small increase of rivalness should be sufficient to deter the consumers from sharing. However, in some particular cases - for example when large network externalities are present on the consumers side, when sharing brings high monetary (through direct payment or advertisement banners) or non-monetary (reputation, glory, self-estime) gains ${ }^{22}$ - the intrinsic gain of sharing, $g_{i}$, can be large. What is more, it takes only one sharing consumer for thousands of others to be able to pirate. As a consequence, the firms will have to adopt more aggressive antipiracy strategies in order to prevent consumers from sharing.

As mentioned in Proposition 1, the consumers will share if and only if the intrinsic gain of sharing, $g_{i}$ exceeds the expected loss due to rivalness, $(1-E) R u_{i}$. Thus, the more valuable the shared good is for the consumer, and the easier it is for the firms to hinder sharing. On the contrary, it will be very difficult for the firms to discourage consumers whose valuation of the good is close to zero from sharing, since these are not harmed when rivalness takes place. It is also worth noticing that anti-piracy strategies aiming at preventing consumers from sharing should be based on an increase of rivalness only. Indeed, any increase of excludability will decrease the expected loss due to rivalness, and thus the impact of an increase of rivalness.

Instead of trying to prevent consumers from sharing, by decreasing $G_{S}$ below zero, firms could target pirating consumers. Propositions 2, 3 and 4 show that consumers do not pirate when the gain of pirating, $G_{P}$, is smaller than the gain of buying, $G_{B}$. The conditions required for $G_{P}>G_{S}$ are detailed in Proposition 2. Depending on the network connectivity, the value of the digital good for the consumers, and the different costs involved in the pirating and buying activities, it is possible to define values for the levels of excludability and rivalness that will be high enough to impede piracy. It is also interesting to observe that these sufficient levels do not usually involve a zero publicness (i.e. $E=R=1$ ). Thus, it is possible for the firms, without reaching a null ex-ante publicness, to achieve a null ex-post publicness.

However, it is not necessarily the case that the anti-piracy strategies of the firms are efficient enough to achieve the required levels of excludability and rivalness. As a consequence, another strategy worth considering consists in decreasing the price of the digital good. Indeed, no consumer will pirate if the price of the digital good is below the total cost of pirating. Unfortunately, although this strategy would probably achieve the targeted goal ${ }^{23}$, firms are usually reluctant to decrease their prices. Nevertheless, such a strategy could be used as a support for the more traditional anti-piracy strategies based on increasing excludability and rivalness. Indeed, from Proposition 2, we can notice

\footnotetext{
${ }^{22}$ See Rayna (2002) for more details on the motivations of sharing consumers.

${ }^{23}$ It is important to notice that the anti-piracy strategies based on increasing excludability and rivalness can be quite costly. Thus, decreasing the price of the digital good instead may not be a worse strategy.
} 
that a strategy involving both a decrease in publicness and a decrease in price is likely to be very efficient, since a decrease in price allows to achieve more easily the levels of excludability and rivalness required to deter piracy. It is also worth noticing that strategies based on increasing excludability should be used with caution since, as discussed above, increasing the excludability can also increase the payoff of sharing and increase the number of consumers sharing the good.

Different anti-piracy strategies are required when mechanisms allowing to encourage, or to force, the cooperation among consumers exist. In this case, as shown in Propositions 5 and 6, an equilibrium involving both sharing and pirating can exist, even when sharing is costly. These forced cooperation mechanisms are not fail-proof however, and Proposition 7 shows that there are some critical values of excludability and rivalness above which the consumers will never choose to pirate (and share). Thus, firms facing pirate networks involving forced cooperation should decrease the ex-ante publicness of the digital good by increasing either the level of excludability, or the level of rivalness, or both. Proposition 7 shows that, if the firms can reach high levels of either excludability or rivalness, it is possible for the firms to adopt a "pure" strategy by increasing only one of these two level. However, if the critical values for excludability and rivalness are out of reach, the firms will need to adopt a "mixed" strategy and to increase both levels. As in the case when forced cooperation does not exist, it is thus possible for the firms to achieve a null ex-post publicness in spite of a positive ex-ante publicness.

It is worthwhile noticing that, in the case of forced cooperation, an increase in the level of rivalness always has a negative impact on the combined profit of pirating and sharing, $G_{P}+G_{S}$. However, this is not necessarily the case for an increase in the excludability level. Indeed, an increase in excludability has two opposite effects on the combined gain of pirating and sharing. As mentioned in Proposition 2, it unambiguously decreases the payoff of pirating. However, as shown in section 3.1, the impact of an increase of excludability can either increase or decrease the payoff of sharing. The overall effect of the impact of an increase of excludability on the combined payoff of pirating and sharing depends on the level of rivalness. If the level of rivalness is rather low, an increase in the level of excludability will lead to a decrease of the combined payoff of pirating and sharing. On the contrary, if the level of rivalness is high, an increase in the level of excludability will lead to a raise of the combined payoff. This is due to the fact that a high level of rivalness leads to a high loss for the sharing consumers. However, when the excludability increases, sharing consumers are less penalised since the other consumers who copied the digital good from them have more chances to be excluded, and therefore rivalness is less likely to take place. These results are detailed in the proposition below:

Proposition 14. The anti-piracy strategies consisting in increasing excludability $(E)$ or rivalness $(R)$ have the following effects on the combined payoff of pirating and sharing, $G_{P}+G_{S}$ :

- An increase in the level of rivalness, $R$, always lead to a decrease in the combined payoff $G_{P}+G_{S}$.

- An increase in the level of excludability, E, can either:

- Lead to a decrease in the combined payoff $G_{P}+G_{S}$ if the level of rivalness, $R$ is below $\frac{N_{i}}{N_{i}+1}$. 
- Lead to an increase in the combined payoff $G_{P}+G_{S}$ if the level of rivalness, $R$ is above $\frac{N_{i}}{N_{i}+1}$.

(Proof given in Appendix)

$$
H\left(G_{P}+G_{S}\right)(E, R)=\left[\begin{array}{cc}
0 & u_{i}\left(1+N_{i}\right) \\
u_{i}\left(1+N_{i}\right) & 0
\end{array}\right]
$$

In addition, from the Hessian matrix of the combined gain $G_{P}+G_{S}$ given by Equation (23), we can notice that the second derivatives are constant and are the same for both the excludability and the rivalness. Thus, both types of strategies - increasing either excludability or rivalness - have the same relative impact. However, as stated above in Proposition 14, increasing excludability only has a positive impact if rivalness is rather low. As it may not be possible for the firms to precisely measure and evaluate the exact levels of excludability and rivalness, it would be advisable for the firms to consider "pure" strategies - e.g. increasing only one of the levels - instead of a mixed one. However, it is also possible that none of the critical values - as defined in Proposition 7 for excludability or rivalness are achievable, in which case the firms will have to consider a mixed strategy in spite of the risk that this strategy might be counter-productive.

The results of Proposition 7 and 14 can also be used when the pirating interactions of the consumers is based on repeated interactions. Indeed, Proposition 10 shows that $G_{P}+G_{S}>G_{B}$ is one of the required conditions of the existence of repeated cooperative pirating strategies among consumers. As such, as in the case of non-repeated interactions with forced cooperation, it is possible for the firms to achieve a null ex-post publicness by adopting policies increasing excludability and/or rivalness.

What is more, Proposition 9 shows that the required discount ratio for a simple cooperative piracy behaviour - where all consumers share and pirate to be sustainable depends positively on the levels of excludability and rivalness. Therefore, the lower the ex-ante publicness is, the more the consumers have to be patient - i.e. the higher the discount ratio has to be - in order for a cooperative piracy strategy to be successful. Nevertheless, when consumers are very patient, Proposition 10 shows that a wide range of cooperative pirating strategies can be devised and sustained by the consumers.

This is particularly important since the firms often use, in addition to strategies aiming at decreasing the publicness of digital goods, other anti-piracy strategies based on the legal system. Indeed, in the countries where piracy and/or sharing is illegal, firms can deter consumers' piracy by monitoring the consumers and suing the offenders for copyright infringement. Alternatively, the firms can also request from the internet service providers that the internet access of the offenders is interrupted. In both cases, the potential loss faced by the offending consumers is expected to be high enough to hinder pirating or sharing behaviours.

In a "perfect world" - where piracy and sharing could always be detected and the offending consumers always prosecuted - this type of strategies would be sufficient to deter sharing, and would thus, even with a total ex-ante publicness, lead to a null ex-post publicness. However, nowadays, it is very unlikely that perfect monitoring exists, and, practically, it is certainly far from being achievable, at least in the near future. In this case, Proposition 10 hints that it is 
very unlikely that firms will be able to create, through an imperfect monitoring, a sufficient deterrent, since the nearly infinite range of supportable cooperative equilibria makes it likely that consumers will be able to avoid detection and punishment. Indeed Proposition 10 shows that sustainable cooperative strategies can include long periods without piracy, or periods when only one consumer pirates, etc.

\subsection{Network structure, anonymity and cooperation}

The results developed in the previous sections show that very different outcomes are obtained depending on the type game considered - e.g. whether the game is repeated or not, or whether forced cooperation exists. This section aims at establishing a link between the type of consumers' network structure - i.e. the type of interactions consumers have between each other - and what we think is the most appropriate model to analyse this structure.

As far as consumers are concerned, the key element regarding their interactions with other consumers certainly lies in the size of the consumers' network. Indeed, in most cases, a larger network means more anonymity for the consumers part of the network: as the network is large, it is more difficult for consumers to identify and "recognise" each other. What is more, as the size of the network is large, the probability of interacting more than once with the same consumer becomes very low.

For these two reasons, in very large networks - which most of the peer-topeer networks (Napster, GNUTella, Kazaa, eMule/eDonkey, BitTorrent) are the interactions between consumers are similar to a non-repeated game, as the one introduced in Section 4.1.1. Thus free-riding among consumers is very likely to occur in these large peer-to-peer networks and indeed most of these networks introduced mechanisms aiming at increasing, or even forcing the cooperation among consumers. Kazaa, for example, uses a priority mechanism based on the sharing history of each consumer of the network. The more a consumer shared in the past, the faster this consumer can access to, and download, digital goods. Consumers who are not sharing can still download digital goods, but it is then likely to take a very long time since they will always be the last ones to be allocated network resources ${ }^{24}$.

Likewise, eMule/eDonkey, BitTorrent, and recent versions of GNUTella, require the consumers to continuously upload while they are downloading ${ }^{25}$. What is more, the consumers who upload with a higher speed - i.e. who share more - are given a higher priority on the network, and are thus able to download faster, which means that they can get more digital goods in the same time than consumers who share less. With this type of mechanism, total free-riding is not feasible, since consumers are forced to share at least a bit in order to get access to the network. However, this usually does not mean that each consumer shares as much as they pirate, and therefore a weaker form of free-riding may exist. As mentioned earlier, a consumer downloading faster than she is uploading will have no incentive to continue sharing after she finishes downloading her copy of

\footnotetext{
${ }^{24}$ They might ultimately have to wait forever, which is equivalent to forbidding them from downloading without sharing.

${ }^{25}$ Usually consumers are only required to share parts of the goods that they are currently downloading. However, there is no requirement that they should share other digital goods.
} 
the digital good. As such she will probably stop sharing before the quantity of digital goods she shared equals the quantity she downloaded.

It is illusory, to expect free-riding to disappear completely in large peer-topeer networks since this would require either a control of the network over the consumers' computers (to prevent consumers from disconnecting before their pirating/sharing ratio is equal to one), or a precise and formal identification of each consumers in order to be able to punish the cheaters. The former is certainly not technically feasible at the moment, while the latter is certainly not desirable since it would also allow the firms to easily identify and sue the infringing consumers.

Nevertheless, Proposition 10 shows that a cooperative strategy could even occur when interactions are rare and spread in time. This means that a repeated game framework can also be used to analyse very large consumers networks. Thus consumers would be likely to pirate and share, and not free-ride at all, as long as they are patient enough, or as long as the pirating game is likely to be repeated. Such situation occurs usually in countries where government policies do not support or enforce IPR: in these countries consumers both pirate and share and little free-riding occurs. However, the situation is usually different in developed countries. Indeed, the law in most of these countries defines higher punishment, for sharing than pirating. This in turns encourages consumers to free-ride and to share as little as possible, while in the meantime consumers try to download as much as possible. Nevertheless, this does not necessarily endangers the viability of the network. Proposition 10 states that a cooperative pirating equilibrium among consumers does not require all consumers to share as much as they pirate. We can thus consider an equilibrium where consumers in countries not enforcing IPR share more than consumers in countries enforcing IPR, and where the situation is more beneficial for all the consumers than if they where buying the digital goods instead.

What is more, Propositions 10 and 11 could be modified in order to take into account the fact that the consumers' payoffs, $G_{P}, G_{S}$ and $G_{B}$, are likely to be different across consumers of one country, and even more across consumers of different countries. In this case, a cooperative equilibrium between consumers in countries enforcing IPR and consumers in countries not enforcing IPR, where consumers in countries not enforcing IPR always share and nearly never pirate (and reciprocally where consumers in countries enforcing IPR pirate and seldom share) can be sustainable. Indeed, we can reasonably assume that in countries not enforcing IPR, the cost of sharing, $G_{S}$, is likely to be close to zero ${ }^{26}$. As such, according to the Corollary of Proposition 11, equilibria where consumers in countries enforcing IPR pirate all the time, and where consumers in countries not enforcing IPR share and virtually never pirate, are sustainable.

In addition to large peer-to-peer networks, consumers are also using smaller scale peer-to-peer networks (e.g. private BitTorrent servers), centralised services (FTP or Hotline servers), or chat services (IRC, MSN, etc.). The smaller size of these networks, their centralised structure, or their interactions at a personal level, make it easier to monitor precisely the contribution of each consumer. For example, pirate FTP servers often contain a bit-for-bit rule preventing the users to download more than they upload. Likewise, private BitTorrent servers

\footnotetext{
${ }^{26}$ In addition, in developing countries, the difference $G_{P}-G_{B}$ is also likely to be larger than in developed countries if the firms are charging the same price in all countries.
} 
often require, as a rule, that each member of the network should maintain a pirating/sharing ratio close to 1 . Failure to comply with this rule usually leads to the consumer being barred from accessing the private server. The interactions among consumers in these networks are very much alike the repeated game developed in Section 5. As such, the Folk theorem developed in Proposition 10 applies, and the consumers in these networks are able, assuming they are patient enough, to develop and sustain many types of cooperative pirating strategies.

\section{Conclusion}

The aim of this article was to investigate the relation between the level of publicness of the digital goods and the pirating and sharing behaviour of the consumers. In order to do so we developed a model - introduced in Section 2 - of pirating and sharing behaviours based on the the determinants of the publicness of digital goods. The main focus of this article was put on the antipiracy strategies of the firms which - by their positive effect on the excludability and the rivalness of the digital good - define the ex-ante level of publicness. The pirating and sharing behaviour of the consumers was then used to discuss the ex-post of publicness of the digital goods.

In Section 3, we shed light upon the pre-conditions, based on the determinants of publicness, which are required for the consumers to pirate and to share. Absolute and relative critical values of network connectivity, excludability and rivalness - such as the consumer never chooses to pirate once these values are reached - are defined. The impact of all the variables on these critical values is then analysed.

A two-player non-repeated version of the model is developed in Section 4 and the possible equilibria characterised. The results obtained show that, both when the game is played simultaneously and sequentially, sub-optimal equilibria may exist when sharing is costly. In this case, the consumers are prone to free-ride among each other and consequently, at the equilibrium, the consumers neither pirate nor share. Provided that the combined payoff of pirating and sharing exceeds the payoff of buying - as it is usually the case nowadays - this situation is sub-optimal. We show that this problem can be solved by introducing a forcedcooperation mechanism, which forces the consumer who pirate to share as well. With such mechanism, the Pareto optimal equilibrium where consumers both pirate and share can be achieved. However, depending on the level of publicness and the other determinants, the consumers can be deterred from pirating and sharing. As we did in Section 3, we define the absolute and relative critical values of network connectivity, excludability and rivalness - such as the consumer never chooses to pirate once these values are reached - when forced cooperation exist. The impact of all the variables on these critical values is also stated.

Section 5 provides a study of an infinitely repeated variant of the model. We first discuss, based on a simple grim-trigger cooperative pirating strategy, the level of discount ratio required for a cooperative equilibrium to be sustainable. The impact of the variables on this critical value of discount ratio is then evaluated. A Folk Theorem, stating all the cooperative sustainable subgame Nash perfect equilibria of the model, is then elaborated. The maximum - and minimum - supportable average SPNE payoffs are stated and the Pareto optimal equilibria are defined. 
The last section of this article is devoted to the analysis of the results obtained in the previous sections. The first part of this section discusses the likely impact of the anti-piracy strategies of the firms depending on the type of pirate network considered. Finally, the question of the network structure and its impact on the anonymity and on the cooperation among consumers is raised.

Overall, we believe that this article gives a good overview of the importance and the role of both ex-ante and ex-post publicness in the pirating and sharing behaviours of the consumers. More importantly, the traditional debate among economists about whether digital goods are private or public goods is brought one step further, since we acknowledge the variability of the publicness of the digital goods and show the impact of this very variability on the consumers' behaviour and on the firms' strategies. Also, our models show that, even in a very homogenous environment, the pirating behaviour of the consumers is likely to be very heterogeneous. Last but not least, this article demonstrates that governments interventions aiming at tackling piracy are - due to imperfect monitoring - very likely to have a small impact on piracy. In addition, the anti-piracy strategies of the firms are usually very costly, and have been unable, so far, to reduce durably the publicness of digital goods. Therefore a decrease in the price of the digital goods seems to be the only remaining option for the digital goods industry.

\section{References}

Abreu, D., March 1988. On the theory of infinitely repeated games with discounting. Econometrica 56 (2), 383-396.

Farrell, J., Maskin, E., December 1989. Renegotiation in repeated games. Games and Economic Behavior 1, 327-360.

Friedman, J. W., Jan. 1971. A non-cooperative equilibrium for supergames. The Review of Economic Studies 38 (1), 1-12.

Fudenberg, D., Levine, D., 1983. Subgame-perfect equilibria of finite and infinite horizon games. Journal of Economic Theory 31, 251-268.

Fudenberg, D., Maskin, E., May 1986. The folk theorem in repeated games with discounting or with incomplete information. Econometrica 54 (3), 533-554.

Fudenberg, D., Tirole, J., 1993. Game Theory. MIT Press.

Pearce, D. G., Dec. 1987. Renegotiation-proof equilibria: Collective rationality and intertemporal cooperation. Cowles Foundation Discussion Papers 855, Cowles Foundation, Yale University, available at http://ideas.repec.org/p/cwl/cwldpp/855.html.

Rayna, T., 2002. Is software a public good? a study of the piracy of digital goods. In: Complexity and the Economy, Implications for Economic Policy. EAEPE Conference 2002.

Rubinstein, A., August 1979. Equilibrium in supergames with the overtaking criterion. Journal of Economic Theory 21 (1), 1-9. 


\section{Appendix}

\section{Proof of Proposition 2}

Preliminary conditions. The consumers will never pirate if the payoff of pirating is smaller than the payoff of buying:

$$
N_{i}(1-E)(1-R) u_{i}-N_{i} c-s \leq u_{i}-p
$$

As the payoff of buying is always postitive $\left(u_{i}>p\right)$, this is the case when:

- The consumer is not connected to any network:

$$
\begin{gathered}
N_{i}=0 \Rightarrow N_{i}(1-E)(1-R) u_{i}-N_{i} c-s=-s \\
s>0 \Rightarrow-s<u_{i}-p
\end{gathered}
$$

- No other consumer is willing to share (equivalent to the result above):

$$
-s<u_{i}-p
$$

Apart from these two rather obvious conditions, it is possible to define some absolute critical values for the environmental variables $E, R, N_{i}$ that will deter the consumer from pirating.

Absolute critical value for network connectivity. Let $N_{i}^{*}$ be the absolute critical value for network connectivity level below which the consumer will never pirate, even when the other conditions are perfect (e.g. $E=0$ and $R=0$ ). When $E=0$ and $R=0$, the payoff of pirating is:

$$
N_{i} u_{i}-N_{i} c-s \leq u_{i}-p \Leftrightarrow N_{i} \leq \frac{u_{i}-p+s}{u_{i}-c}
$$

Thus:

$$
N_{i}^{*}=\frac{u_{i}-p+s}{u_{i}-c}
$$

And when $N_{i}<N_{i}^{*}$ the consumer will never pirate $\forall E, R \in[0,1]$

Absolute critical value for excludability. Let $E^{*}$ be the absolute critical value for excludability level above which the consumer will never pirate, even when the other conditions are perfect (e.g. $N_{i}=1$ and $R=0$ ). When $N_{i}=1$ and $R=0$, the payoff of pirating is:

$$
(1-E) u_{i}-c-s \leq u_{i}-p \Leftrightarrow E \geq \frac{p-s-c}{u_{i}}
$$

Thus:

$$
E^{*}=\frac{p-s-c}{u_{i}}
$$

And when $E>E^{*}$ the consumer will never pirate $\forall N_{i}, R \in[0,1]$ 
Absolute critical value for rivalness. Using the same method as above, we show that the absolute critical value for rivalness is:

$$
R^{*}=\frac{p-s-c}{u_{i}}
$$

And when $R>R^{*}$ the consumer will never pirate $\forall N_{i}, E \in[0,1]$

If the absolute critical for network connectivity, $N_{i}^{*}$ is not reached, it is possible to define relative critical values for excludability and rivalness based on the value of the network connectivity.

Relative critical value for excludability as a function of $N_{i}$. Let $E^{*}\left(N_{i}\right)$ be the relative critical value for excludability level, based on the current network connectivity, above which the consumer will never pirate, even when the rivalness is null $(R=0)$. When $R=0$, the payoff of pirating is:

$$
N_{i}(1-E) u_{i}-N_{i} c-s \leq u_{i}-p \Leftrightarrow E \geq \frac{p-s-N_{i} c-\left(1-N_{i}\right) u_{i}}{N_{i} u_{i}}
$$

Thus:

$$
E^{*}\left(N_{i}\right)=\frac{p-s-N_{i} c-\left(1-N_{i}\right) u_{i}}{N_{i} u_{i}}
$$

And when $E>E^{*}\left(N_{i}\right)$ the consumer will never pirate $\forall R \in[0,1]$

Relative critical value for rivalness as a function of $N_{i}$. Using the same method as above, we show that the relative critical value for rivalness as a function of network connectivity is:

$$
R^{*}\left(N_{i}\right)=\frac{p-s-N_{i} c-\left(1-N_{i}\right) u_{i}}{N_{i} u_{i}}
$$

And when $R>R^{*}\left(N_{i}\right)$ the consumer will never pirate $\forall E \in[0,1]$

If none of the relative critical values defined above are reached, it is possible to define relative critical values for excludability and rivalness based on oneanother and on the value of the network connectivity.

Relative critical value for excludability as a function of $N_{i}$ and $R . \quad E^{*}\left(N_{i}, R\right)$ is the relative critical value for excludability level, based on the current network connectivity and on the current level of rivalness, above which the consumer will never pirate:

$$
N_{i}(1-E)(1-R) u_{i}-N_{i} c-s \leq u_{i}-p \Leftrightarrow E \geq \frac{p-s-N_{i} c-\left(1-N_{i}(1-R)\right) u_{i}}{N(1-R) u_{i}}
$$

Thus:

$$
E^{*}\left(N_{i}, R\right)=\frac{p-s-N_{i} c-\left(1-N_{i}(1-R)\right) u_{i}}{N(1-R) u_{i}}
$$

And when $E>E^{*}\left(N_{i}, R\right)$ the consumer will never pirate. 
Relative critical value for rivalness as a function of $N_{i}$ and $E$. Using the same method as above, we show that the relative critical value for rivalness as a function of network connectivity is:

$$
R^{*}\left(N_{i}, E\right)=\frac{p-s-N_{i} c-\left(1-N_{i}(1-E)\right) u_{i}}{N(1-E) u_{i}}
$$

And when $R>R^{*}\left(N_{i}, E\right)$ the consumer will never pirate.

Corollary (1).

$$
\frac{\partial N_{i}^{*}}{\partial u_{i}}=\frac{p-s-c}{\left(u_{i}-c\right)^{2}}>0
$$

(By assumption, $p>s+c$ )

$$
\frac{\partial N_{i}^{*}}{\partial p}=-\frac{1}{u_{i}-c}<0
$$

(By assumption, $u>c$ )

$$
\begin{gathered}
\frac{\partial N_{i}^{*}}{\partial s}=\frac{1}{u_{i}-c}>0 \\
\frac{\partial N_{i}^{*}}{\partial c}=-\frac{p-s-c}{\left(u_{i}-c\right)^{2}}<0 \\
\frac{\partial E^{*}}{\partial u_{i}}=\frac{\partial R^{*}}{\partial u_{i}}=-\frac{p-s-c}{\left(u_{i}\right)^{2}}<0 \\
\frac{\partial E^{*}}{\partial p}=\frac{\partial R^{*}}{\partial p}=\frac{1}{\left(u_{i}\right)}>0 \\
\frac{\partial E^{*}}{\partial s}=\frac{\partial R^{*}}{\partial s}=-\frac{1}{\left(u_{i}\right)}<0 \\
\frac{\partial E^{*}}{\partial c}=\frac{\partial R^{*}}{\partial c}=-\frac{1}{\left(u_{i}\right)}<0
\end{gathered}
$$

Similar calculations show that $E^{*}\left(N_{i}\right), E^{*}\left(N_{i}, R\right), R^{*}\left(N_{i}\right)$ and $R^{*}\left(N_{i}, E\right)$ evolve in the same way as $E^{*}$ and $R^{*}$ do.

Corollary (2).

$$
\begin{gathered}
\frac{\partial E^{*}\left(N_{i}, R\right)}{\partial N_{i}}=\frac{u_{i}+s-p}{N_{i}^{2}(1-R) u_{i}}>0 \\
\frac{\partial E^{*}\left(N_{i}, R\right)}{\partial R}=-\frac{u_{i}+s-p}{N_{i}^{2}(1-R) u_{i}}<0 \\
\frac{\partial R^{*}\left(N_{i}, E\right)}{\partial N_{i}}=\frac{u_{i}+s-p}{N_{i}^{2}(1-E) u_{i}}>0 \\
\frac{\partial R^{*}\left(N_{i}, E\right)}{\partial E}=-\frac{u_{i}+s-p}{N_{i}^{2}(1-E) u_{i}}<0
\end{gathered}
$$




\section{Proof of Proposition 7}

When a forced cooperation mechanism exists, the consumers will never pirate if the combined payoff of pirating and sharing, $G_{P}+G_{S}$ is smaller than the payoff of buying, $G_{B}$. This is the case when:

$$
N_{i}(1-E)(1-R) u_{i}-N_{i} c-s+g_{i}-(1-E) R u_{i}<u_{i}-p
$$

Intrinsic benefit/cost of sharing. A first reason for the consumer not to pirate is when the benefit/cost of sharing, $g_{i}$ is so (negatively) low that pirating and sharing is never the best option, even when the environmental conditions are perfect-e.g. $N_{i}=1, E=0, R=0$. When $N_{i}=1, E=0, R=0$, the combined payoff of pirating and sharing is:

$$
u_{i}-c-s+g_{i}<u_{i}-p \Leftrightarrow g_{i}<-p+s+c
$$

When $g_{i}<-p+s+c$, the consumer will never pirate and share $\forall\left\{N_{i}, E, R\right\} \in$ $[0,1]^{3}$

Absolute critical value of network connectivity. $N_{i}^{* *}$ is the absolute value of network connectivity, when there is forced cooperation, below which the consumer will never choose to pirate and share, even when the other environmental conditions are perfect - i.e. $E=0, R=0$. In this case, the combined payoff of pirating and sharing is:

$$
N_{i} u_{i}-N_{i} c-s+g_{i}<u_{i}-p \Leftrightarrow N_{i}<\frac{u_{i}-p+s-g_{i}}{u_{i}-c}
$$

When $N_{i}<N_{i}^{* *}=\frac{u_{i}-p+s-g_{i}}{u_{i}-c}$, the consumer will never pirate and share for any $\{E, R\} \in[0,1]$

If the absolute critical for network connectivity, $N_{i}^{* *}$ is not reached, it is possible to define relative critical values for excludability and rivalness based on the value of the network connectivity.

Relative critical value for excludability as a function of $N_{i}$. Let $E^{* *}\left(N_{i}\right)$ be the relative critical value for excludability level, based on the current network connectivity, above which the consumer will never pirate, even when the rivalness is null $(R=0)$. When $R=0$, the payoff of pirating is:

$$
N_{i}(1-E) u_{i}-N_{i} c-s+g_{i}<u_{i}-p \Leftrightarrow E>\frac{p-s-N_{i} c-\left(1-N_{i}\right) u_{i}+g_{i}}{N_{i} u_{i}}
$$

Thus:

$$
E^{* *}\left(N_{i}\right)=\frac{p-s-N_{i} c-\left(1-N_{i}\right) u_{i}+g_{i}}{N_{i} u_{i}}
$$

And when $E>E^{* *}\left(N_{i}\right)$ the consumer will never pirate $\forall R \in[0,1]$

Relative critical value for rivalness as a function of $N_{i}$. Using the same method as above, we show that the relative critical value for rivalness as a function of network connectivity is:

$$
R^{* *}\left(N_{i}\right)=\frac{p-s-N_{i} c-\left(1-N_{i}\right) u_{i}+g_{i}}{N_{i} u_{i}}
$$

And when $R>R^{* *}\left(N_{i}\right)$ the consumer will never pirate $\forall E \in[0,1]$ 
If none of the relative critical values defined above are reached, it is possible to define relative critical values for excludability and rivalness based on oneanother and on the value of the network connectivity.

Relative critical value for excludability as a function of $N_{i}$ and $R . E^{* *}\left(N_{i}, R\right)$ is the relative critical value for excludability level, based on the current network connectivity and on the current level of rivalness, above which the consumer will never pirate:

$$
\begin{gathered}
N_{i}(1-E)(1-R) u_{i}-N_{i} c-s+g_{i}-(1-E) R u_{i}<u_{i}-p \\
\Leftrightarrow E>\frac{p-s-N_{i} c+g_{i}-\left(1-N_{i}(1-R)+R\right) u_{i}}{\left(N_{i}(1-R)-R\right) u_{i}}
\end{gathered}
$$

Thus:

$$
E^{* *}\left(N_{i}, R\right)=\frac{p-s-N_{i} c+g_{i}-\left(1-N_{i}(1-R)+R\right) u_{i}}{\left(N_{i}(1-R)-R\right) u_{i}}
$$

And when $E>E^{* *}\left(N_{i}, R\right)$ the consumer will never pirate.

Relative critical value for rivalness as a function of $N_{i}$ and $E$. Using the same method as above, we show that the relative critical value for rivalness as a function of network connectivity is:

$$
R^{* *}\left(N_{i}, E\right)=\frac{p-s-N_{i} c+g_{i}-\left(1-N_{i}(1-E)+E\right) u_{i}}{\left(N_{i}(1-E)-E\right) u_{i}}
$$

And when $R>R^{* *}\left(N_{i}, E\right)$ the consumer will never pirate.

Corollary (1). Assuming that $g_{i}<0$ and $0>g_{i}>-p+s+c \Leftrightarrow p-s-c+g_{i}>0$

$$
\begin{aligned}
& \frac{\partial N_{i}^{* *}}{\partial u_{i}}=\frac{p-s-c+g_{i}}{\left(u_{i}-c\right)^{2}}>0 \\
& \frac{\partial N_{i}^{* *}}{\partial p}=-\frac{1}{u_{i}-c}<0 \\
& \frac{\partial N_{i}^{* *}}{\partial s}=\frac{1}{u_{i}-c}>0 \\
& \frac{\partial N_{i}^{* *}}{\partial c}=\frac{u_{i}-p+s-g_{i}}{\left(u_{i}-c\right)^{2}}>0 \\
& \frac{\partial N_{i}^{* *}}{\partial g_{i}}=-\frac{1}{u_{i}-c}<0 \\
& \frac{\partial E^{* *}\left(N_{i}\right)}{\partial u_{i}}=\frac{\partial R^{* *}\left(N_{i}\right)}{\partial u_{i}}=\frac{-p+s+N_{i} c-g_{i}}{\left(N_{i} u_{i}\right)^{2}}<0 \\
& \frac{\partial E^{* *}\left(N_{i}\right)}{\partial p}=\frac{\partial R^{* *}\left(N_{i}\right)}{\partial p}=\frac{1}{\left(N_{i} u_{i}\right)}>0 \\
& \frac{\partial E^{* *}\left(N_{i}\right)}{\partial s}=\frac{\partial R^{* *}\left(N_{i}\right)}{\partial s}=-\frac{1}{\left(N_{i} u_{i}\right)}<0 \\
& \frac{\partial E^{* *}\left(N_{i}\right)}{\partial c}=\frac{\partial R^{* *}\left(N_{i}\right)}{\partial c}=-\frac{1}{\left(u_{i}\right)}<0 \\
& \frac{\partial E^{* *}\left(N_{i}\right)}{\partial g_{i}}=\frac{\partial R^{* *}\left(N_{i}\right)}{\partial g_{i}}=\frac{1}{\left(N_{i} u_{i}\right)}>0
\end{aligned}
$$


Similar calculations show that $E^{* *}\left(N_{i}, R\right)$ and $R^{* *}\left(N_{i}, E\right)$ evolve in the same way as $E^{* *}\left(N_{i}\right)$ and $R^{* *}\left(N_{i}\right)$ do.

Corollary (2). Given that $u_{i}>p$ and $g_{i}<0$ :

$$
\begin{aligned}
& \frac{\partial E^{* *}\left(N_{i}, R\right)}{\partial N_{i}}=\frac{\left.(1-R)\left(u-p+s-g_{i}\right)+c R\right)}{\left(R-(1-R) N_{i}\right)^{2} u_{i}}>0 \\
& \frac{\partial E^{* *}\left(N_{i}, R\right)}{\partial R}=-\frac{\left(1+N_{i}\right)\left(u_{i}-p+s+N_{i} c-g_{i}\right)}{\left(R-(1-R) N_{i}\right)^{2} u_{i}}<0 \\
& \frac{\partial R^{* *}\left(N_{i}, E\right)}{\partial N_{i}}=\frac{\left.(1-E)\left(u-p+s-g_{i}\right)+c E\right)}{\left(E-(1-E) N_{i}\right)^{2} u_{i}}>0 \\
& \frac{\partial R^{* *}\left(N_{i}, E\right)}{\partial E}=-\frac{\left(1+N_{i}\right)\left(u_{i}-p+s+N_{i} c-g_{i}\right)}{\left(E-(1-E) N_{i}\right)^{2} u_{i}}<0
\end{aligned}
$$

\section{Proof of Corollaries 1 and 2 of Proposition 9}

Impact of utility.

$$
\begin{gathered}
\frac{\partial \delta^{*}}{\partial u_{i}}=\frac{-\partial_{u_{i}} G_{S}\left(G_{P}-G_{B}\right)-\left(-G_{S}\right) \partial_{u_{i}}\left(G_{P}-G_{B}\right)}{\left(G_{P}-G_{B}\right)^{2}} \\
-\partial_{u_{i}} G_{S}=(1-E) R \geq 0
\end{gathered}
$$

By assumption, $G_{P} \geq G_{B}$. Thus:

$$
\begin{gathered}
-\partial_{u_{i}} G_{S}\left(G_{P}-G_{B}\right) \geq 0 \\
\partial_{u_{i}}\left(G_{P}-G_{B}\right)=-1+(1-E) N(1-R) \leq 0
\end{gathered}
$$

By assumption, $G_{S}<0$. Thus:

$$
-\left(-G_{S}\right) \partial_{u_{i}}\left(G_{P}-G_{B}\right) \geq 0
$$

Therefore, $-\partial_{u_{i}} G_{S}\left(G_{P}-G_{B}\right)-\left(-G_{S}\right) \partial_{u_{i}}\left(G_{P}-G_{B}\right) \geq 0$ and:

$$
\frac{\partial \delta^{*}}{\partial u_{i}} \geq 0
$$

Impact of the search costs.

$$
\begin{gathered}
\frac{\partial \delta^{*}}{\partial s}=\frac{-\partial_{s} G_{S}\left(G_{P}-G_{B}\right)-\left(-G_{S}\right) \partial_{s}\left(G_{P}-G_{B}\right)}{\left(G_{P}-G_{B}\right)^{2}} \\
-\partial_{s} G_{S}=0 \\
\partial_{s}\left(G_{P}-G_{B}\right)=-1
\end{gathered}
$$

By assumption, $G_{S}<0$. Thus:

$$
-\left(-G_{S}\right) \partial_{s}\left(G_{P}-G_{B}\right)=-G_{S} \geq 0
$$

Therefore, $-\partial_{s} G_{S}\left(G_{P}-G_{B}\right)-\left(-G_{S}\right) \partial_{s}\left(G_{P}-G_{B}\right) \geq 0$ and:

$$
\frac{\partial \delta^{*}}{\partial s} \geq 0
$$


Impact of the copy cost.

$$
\begin{gathered}
\frac{\partial \delta^{*}}{\partial c}=\frac{-\partial_{c} G_{S}\left(G_{P}-G_{B}\right)-\left(-G_{S}\right) \partial_{c}\left(G_{P}-G_{B}\right)}{\left(G_{P}-G_{B}\right)^{2}} \\
-\partial_{c} G_{S}=0 \\
\partial_{c}\left(G_{P}-G_{B}\right)=-N_{i}
\end{gathered}
$$

By assumption, $G_{S}<0$. Thus:

$$
-\left(-G_{S}\right) \partial_{c}\left(G_{P}-G_{B}\right)=-N_{i} G_{S} \geq 0
$$

Therefore, $-\partial_{c} G_{S}\left(G_{P}-G_{B}\right)-\left(-G_{S}\right) \partial_{c}\left(G_{P}-G_{B}\right) \geq 0$ and:

$$
\frac{\partial \delta^{*}}{\partial c} \geq 0
$$

Impact of the price.

$$
\begin{gathered}
\frac{\partial \delta^{*}}{\partial p}=\frac{-\partial_{p} G_{S}\left(G_{P}-G_{B}\right)-\left(-G_{S}\right) \partial_{p}\left(G_{P}-G_{B}\right)}{\left(G_{P}-G_{B}\right)^{2}} \\
-\partial_{p} G_{S}=0 \\
\partial_{p}\left(G_{P}-G_{B}\right)=1
\end{gathered}
$$

By assumption, $G_{S}<0$. Thus:

$$
-\left(-G_{S}\right) \partial_{p}\left(G_{P}-G_{B}\right)=G_{S}<0
$$

Therefore, $-\partial_{p} G_{S}\left(G_{P}-G_{B}\right)-\left(-G_{S}\right) \partial_{p}\left(G_{P}-G_{B}\right)<0$ and:

$$
\frac{\partial \delta^{*}}{\partial p}<0
$$

Impact of the sharing cost.

$$
\begin{gathered}
\frac{\partial \delta^{*}}{\partial g}=\frac{-\partial_{g} G_{S}\left(G_{P}-G_{B}\right)-\left(-G_{S}\right) \partial_{g}\left(G_{P}-G_{B}\right)}{\left(G_{P}-G_{B}\right)^{2}} \\
-\partial_{g} G_{S}=-1
\end{gathered}
$$

By assumption, $G_{P} \geq G_{B}$. Thus:

$$
\begin{gathered}
-\partial_{g} G_{S}\left(G_{P}-G_{B}\right)<0 \\
\partial_{g}\left(G_{P}-G_{B}\right)=0
\end{gathered}
$$

Therefore, $-\partial_{g} G_{S}\left(G_{P}-G_{B}\right)-\left(-G_{S}\right) \partial_{g}\left(G_{P}-G_{B}\right)<0$ and:

$$
\frac{\partial \delta^{*}}{\partial g}<0
$$


Impact of the excludability.

$$
\begin{gathered}
\frac{\partial \delta^{*}}{\partial E}=\frac{-\partial_{E} G_{S}\left(G_{P}-G_{B}\right)-\left(-G_{S}\right) \partial_{E}\left(G_{P}-G_{B}\right)}{\left(G_{P}-G_{B}\right)^{2}} \\
-\partial_{E} G_{S}\left(G_{P}-G_{B}\right)-\left(-G_{S}\right) \partial_{E}\left(G_{P}-G_{B}\right)=u_{i}\left(R\left(u_{i}+s+N_{i} c-p\right)\right. \\
\left.-N_{i} g_{i}(1-R)\right) \\
\frac{\partial \delta^{*}}{\partial E}>0 \Leftrightarrow R\left(u_{i}+s+N_{i} c-p\right)-N_{i} g(1-R)>0
\end{gathered}
$$

By assumption, $u_{i}>p$, thus:

$$
R\left(u_{i}+s+N_{i} c-p\right)>0
$$

By assumption, $g_{i}<0$, thus:

$$
-N_{i} g(1-R)>0
$$

Therefore $R\left(u_{i}+s+N_{i} c-p\right)-N_{i} g(1-R)>0$ and:

$$
\frac{\partial \delta^{*}}{\partial E}>0
$$

Impact of the rivalness.

$$
\begin{gathered}
\frac{\partial \delta^{*}}{\partial R}=\frac{-\partial_{R} G_{S}\left(G_{P}-G_{B}\right)-\left(-G_{S}\right) \partial_{R}\left(G_{P}-G_{B}\right)}{\left(G_{P}-G_{B}\right)^{2}} \\
-\partial_{R} G_{S}=(1-E) u_{i}>0
\end{gathered}
$$

By assumption, $G_{P}>G_{B}$. Thus:

$$
\begin{gathered}
-\partial_{R} G_{S}\left(G_{P}-G_{B}\right)>0 \\
\partial_{R}\left(G_{P}-G_{B}\right)=-(1-E) N_{i} u_{i}<0
\end{gathered}
$$

By assumption, $G_{S}<0$. Thus:

$$
-\left(-G_{S}\right) \partial_{R}\left(G_{P}-G_{B}\right)>0
$$

Therefore, $-\partial_{R} G_{S}\left(G_{P}-G_{B}\right)-\left(-G_{S}\right) \partial_{R}\left(G_{P}-G_{B}\right)>0$ and:

$$
\frac{\partial \delta^{*}}{\partial R}>0
$$

Impact of network connectivity.

$$
\frac{\partial \delta^{*}}{\partial N_{i}}=\frac{-\partial_{N_{i}} G_{S}\left(G_{P}-G_{B}\right)-\left(-G_{S}\right) \partial_{N_{i}}\left(G_{P}-G_{B}\right)}{\left(G_{P}-G_{B}\right)^{2}}
$$


$-\partial_{N_{i}} G_{S}\left(G_{P}-G_{B}\right)-\left(-G_{S}\right) \partial_{N_{i}}\left(G_{P}-G_{B}\right)=\left((1-E)(1-R) u_{i}-c\right)\left(g-(1-E) R u_{i}\right)$

$\frac{\partial \delta^{*}}{\partial N_{i}}>0$ if and only if:

$$
(1-E)(1-R) u_{i}-c>0 \text { and } g-(1-E) R u_{i}>0
$$

Or:

$$
(1-E)(1-R) u_{i}-c<0 \text { and } g-(1-E) R u_{i}<0
$$

Under the usual assumptions, $g-(1-E) R u_{i}<0$, and:

$$
(1-E)(1-R) u_{i}-c<0 \Leftrightarrow(1-E)(1-R) u_{i}<c
$$

As this would mean that the expected utility of the good, $(1-E)(1-R) u_{i}$ is lower than the cost of copying, $c$, we assume that this is not the case, as otherwise the consumer would certainly not choose to pirate.

As we assume that $(1-E)(1-R) u_{i}>c$ :

$$
\left((1-E)(1-R) u_{i}-c\right)\left(g-(1-E) R u_{i}\right)<0
$$

and:

$$
\frac{\partial \delta^{*}}{\partial N_{i}}<0
$$

\section{Proof of Proposition 10}

The Folk Theorem. Let $\hat{\sigma}_{i}$ be a strategy leading to the discounted average payoff $\hat{\nu}_{i}^{\infty}$ greater than the minmax payoff $G_{B}$ for all player $i$. Specify the following behaviour: each players $i$ plays the strategy $\hat{\sigma}_{i}$ as long as all the players have played $\hat{\sigma}_{i}$ before. All players use the Nash reversion strategy: if someone has deviated in the past, all the players play the safe strategy $\sigma^{s}$ forever.

Due to the Nash reversion strategy, a player defecting at time $t=k$ gains at most a one-time bounded payoff $C_{t}^{27}$, but looses the gain from future cooperation equal to:

$$
\left(\hat{\nu}_{i}^{\infty}-G_{B}\right)\left(\sum_{t=k}^{\infty} \delta^{t-k+1}\right)
$$

and:

$$
\lim _{\delta \rightarrow 1}\left[\left(\hat{\nu}_{i}^{\infty}-G_{B}\right)\left(\sum_{t=k}^{\infty} \delta^{t-k+1}\right)\right]=\infty
$$

Thus, there exist a $\underline{\delta}<1$ such as, for $t=k$ :

$$
\left(\hat{\nu}_{i}^{\infty}-G_{B}\right)\left(\sum_{t=k}^{\infty} \underline{\delta}^{t-k+1}\right)>C_{t}
$$

\footnotetext{
${ }^{27}$ in this particular game, the defecting gain is at most $G_{P}$.
} 
Corollary 1. A pair of strategies $\left(\sigma_{i}, \sigma_{i}\right)$ for all players $i=1,2$, involving one episode of cooperative piracy, when both consumers pirate and share during the same period and get a payoff of $G_{P}+G_{S}$, and the buying action giving the minmax payoff $G_{B}$ otherwise, has for discounted average payoff:

$$
\hat{\nu}_{i}^{\infty}=(1-\delta)\left(G_{B}+\delta G_{B}+\ldots+\delta^{k}\left(G_{P}+G_{S}\right)+\ldots+\delta^{n} G_{B}+\ldots\right)
$$

As long as $G_{P}+G_{S}>G_{B}$,

$$
\hat{\nu}_{i}^{\infty}>G_{B}
$$

Thus, following the Folk Theorem, for all $\delta$ sufficiently close to one, $\left(\sigma_{i}, \sigma_{i}\right)$ is a SPNE. By extension, every pair of strategies involving more than one episode of cooperative piracy is a SPNE when $\delta$ is sufficiently close to one.

Corollary 2. Let $\left(\sigma_{1}, \sigma_{2}\right)$ be a pair of strategies consisting in infinitely repeated sequences of actions ${ }^{28}$. Each sequence lasts for $T_{1}$ periods and includes one episode of planned reciprocal cooperative defection and buying actions otherwise. A planned reciprocal cooperative defection episode is defined as follows: one of the player pirates without sharing and gets a payoff equal to $G_{P}$; as this is a planned defection, the other player shares and chooses not to pirate and gets a payoff equal to $G_{B}+G_{S}$; each player then does the opposite action during the next period. The average discounted payoff for this sequence of actions for each player is then:

$$
\begin{aligned}
& \hat{\nu}_{1}^{T_{1}}=(1-\delta)\left(G_{B}+\delta G_{B}+\ldots+\delta^{k}\left(G_{P}\right)+\delta^{k+1}\left(G_{B}+G_{S}\right)+\ldots+\delta^{T 1}\right) \\
& \hat{\nu}_{2}^{T_{1}}=(1-\delta)\left(G_{B}+\delta G_{B}+\ldots+\delta^{k}\left(G_{B}+G_{S}\right)+\delta^{k+1}\left(G_{P}\right)+\ldots+\delta^{T 1}\right)
\end{aligned}
$$

As long as $G_{P}+G_{S}>G_{B}$,

$$
\begin{aligned}
& \hat{\nu}_{1}^{T_{1}}>G_{B} \\
& \hat{\nu}_{2}^{T_{1}}>G_{B}
\end{aligned}
$$

If this sequences are repeated infinitely, the average discounted payoff for each player is:

$$
\begin{aligned}
& \hat{\nu}_{1}^{\infty}=\hat{\nu}_{1}^{T_{1}}+\delta^{T 1} \hat{\nu}_{1}^{T_{1}}+\ldots \\
& \hat{\nu}_{2}^{\infty}=\hat{\nu}_{2}^{T_{1}}+\delta^{T 1} \hat{\nu}_{2}^{T_{1}}+\ldots
\end{aligned}
$$

And:

$$
\begin{aligned}
& \hat{\nu}_{1}^{\infty}>G_{B} \\
& \hat{\nu}_{2}^{\infty}>G_{B}
\end{aligned}
$$

Thus, following the Folk Theorem, for all $\delta$ sufficiently close to one, $\left(\sigma_{1}, \sigma_{2}\right)$ is a SPNE. By extension, every pair of strategies involving sequences with more than one episode of planned reciprocal cooperative defection is a SPNE when $\delta$ is sufficiently close to one.

\footnotetext{
${ }^{28} \mathrm{~A}$ similar type of demonstration, involving repeated sequences of actions, can also be used to prove Corollary (1). When the duration of the sequence $T_{1}$ is large $\left(T_{1} \rightarrow \infty\right)$, both methods of demonstration are in fact equivalent.
} 


\section{Proof of Proposition 11}

Maximum supportable SPNE average payoff. The theoretical maximum average discounted payoff that can be achieved by a player $i, \hat{\nu}_{i}^{\max T}$, equal to the payoff of pirating $G_{P}$, since that is when the consumer pirates all the time without sharing that she gets the highest payoff:

$$
\hat{\nu}_{i}^{\max T}=G_{P}
$$

However, this payoff $\hat{\nu}_{i}^{\operatorname{maxT}}$ can be a supportable average SPNE payoff if and only if the corresponding average discounted payoff of the other player is at least equal to the reservation utility $G_{B}$. For one of the player to get an average discounted payoff equal to $\hat{\nu}_{i}^{\max T}$, the other player should share without pirating, and thus would get an average discounted payoff equal to the combined payoff of buying and sharing: $G_{B}+G_{S}$. Thus, as long as sharing is costly, the theoretical maximum average discounted payoff $\hat{\nu}_{i}^{\max T}$ can not be the maximum supportable SPNE average payoff as the corresponding average discounted payoff for the other player is in this case lower than the reservation utility:

$$
G_{S}<0 \Rightarrow \hat{\nu}_{i}^{\max }<\nu_{i}^{\max T}
$$

Let the two players $i=1,2$ play a mixed strategy where the pure strategy $P S$ - pirate and share - is played with the probability $\lambda_{i}$ and the pure strategy $\bar{P} S$ - buy and share - is played with the probability $\left(1-\lambda_{i}\right)$. The expected average discounted payoff of player $i$ is then:

$$
\hat{\nu}_{i}\left(\lambda_{i}\right)=\lambda_{i} G_{P}+\left(1-\lambda_{i}\right) G_{B}+\lambda_{j} G_{S}
$$

In order to find the maximum supportable SPNE average payoff, let's assume that, aiming at reaching the highest possible payoff, player 1 chooses $\lambda_{1}=1$ and always pirates and shares. The highest payoff for player 1 will be reached when player 2 chooses the smallest possible $\lambda_{2}-$ e.g. shares without pirating as often as possible - under the constraint that the the average discounted payoff for player 2 is higher than the reservation utility:

$$
\hat{\nu}_{2}\left(\lambda_{2}\right) \geq G_{B} \Leftrightarrow \lambda_{2} \geq \frac{-\lambda_{1} G_{S}}{G_{P}-G_{S}}
$$

Thus, the smallest possible value of $\lambda_{i}$ leading to a discounted average payoff equal to the reservation utility for player $i$, and to the highest possible average discounted payoff for player $j$ is:

$$
\lambda_{i}^{\min }\left(\lambda_{j}\right)=\frac{-\lambda_{j} G_{S}}{G_{P}-G_{S}}
$$

Accordingly, if player 1 plays $\lambda_{1}=1$ and if player 2 plays $\lambda_{2}=\lambda_{2}^{\min }(1)=$, the payoff of player 1 is:

$$
\hat{\nu}_{1}=G_{P}+\frac{-G_{S}}{G_{P}-G_{S}} G_{S}=G_{P}-\frac{G_{S}{ }^{2}}{G_{P}-G_{S}}
$$

Therefore, the maximum supportable SPNE average payoff for player $i$ is:

$$
\hat{\nu}_{i}^{\max }=G_{P}-\frac{G_{S}{ }^{2}}{G_{P}-G_{S}}
$$


This maximum supportable SPNE average payoff is achieved when $\lambda_{i}=1$ and $\lambda_{j}=\frac{-G_{S}}{G_{P}-G_{S}}$.

Corollary. The maximum supportable SPNE average discounted payoff, $\hat{\nu}_{i}^{\max }$ goes to $\hat{\nu}_{i}^{\max T}=G_{P}$ when the cost of sharing becomes very small or when the difference between the payoff of pirating and the payoff of buying becomes large:

$$
\begin{gathered}
\lim _{G_{S} \rightarrow 0} \hat{\nu}_{i}^{\max }=G_{P}=\hat{\nu}_{i}^{\max T} \\
\lim _{\left(G_{B}-G_{P}\right) \rightarrow \infty} \hat{\nu}_{i}^{\max }=G_{P}=\hat{\nu}_{i}^{\max T}
\end{gathered}
$$

\section{Proof of Proposition 12}

Pareto Optimality. $\left(\sigma_{i}, \sigma_{j}\right)$ is a pair of strategies such that each player $i=1,2$ plays $P S$ with probability $\lambda_{i}$ and $\bar{P} S$ with probability $\left(1-\lambda_{i}\right)$ and $\lambda_{i}=1$ and $\lambda_{j} \in[0,1]$ or $\lambda_{i} \in[0,1]$ and $\lambda_{j}=1$. $\left(\hat{\nu}_{i}, \hat{\nu}_{j}\right)$ are the average discounted payoffs resulting from these pair of strategies. $\left(\hat{\nu}_{i}, \hat{\nu}_{j}\right)$ are Pareto optimal payoffs if no other payoffs $\left(\hat{\nu}_{i}^{\prime}, \hat{\nu}_{j}^{\prime}\right)$ such as:

$$
\left\{\begin{array}{lll}
\hat{\nu}_{i}^{\prime}>\hat{\nu}_{i} & \text { and } & \hat{\nu}_{j}^{\prime} \geq \hat{\nu}_{j} \\
& \text { or } & \\
\hat{\nu}_{i}^{\prime} \geq \hat{\nu}_{i} & \text { and } & \hat{\nu}_{j}^{\prime}>\hat{\nu}_{j}
\end{array}\right.
$$

Let $\left(\lambda_{i}^{\prime}, \lambda_{j}^{\prime}\right)$ be the mixed strategies leading to the payoffs $\left(\hat{\nu}_{i}^{\prime}, \hat{\nu}_{j}^{\prime}\right)$. From Equation (A2):

$$
\begin{gathered}
\hat{\nu}_{i}=\lambda_{i} G_{P}+\left(1-\lambda_{i}\right) G_{B}+\lambda_{j} G_{S} \\
\hat{\nu}_{i}^{\prime}=\lambda_{i}^{\prime} G_{P}+\left(1-\lambda_{i}\right) G_{B}+\lambda_{j}^{\prime} G_{S} \\
\hat{\nu}_{i}^{\prime}>\hat{\nu}_{i} \Leftrightarrow\left\{\begin{array}{ccc}
\lambda_{i}^{\prime} & >\lambda_{i} \\
& \text { or } \\
\lambda_{j}^{\prime} & <\lambda_{j}
\end{array}\right.
\end{gathered}
$$

When $\lambda_{i}=1$ and $\lambda_{j} \in[0,1], \lambda_{i}^{\prime} \leq \lambda_{i}$. Thus:

$$
\hat{\nu}_{i}^{\prime}>\hat{\nu}_{i} \Leftrightarrow\left\{\begin{array}{ccc}
\lambda_{i}^{\prime} & =\lambda_{i}=1 \\
& \text { and } \\
\lambda_{j}^{\prime} & <\lambda_{j}
\end{array}\right.
$$

If $\lambda_{j}^{\prime}<\lambda_{j}$,

$$
\hat{\nu}_{j}^{\prime}=\hat{\nu}_{j} \Leftrightarrow \lambda_{i}^{\prime}<\lambda_{i}
$$

Equations (A5) and (A6) are mutually exclusive. Thus, there are no $\left(\lambda_{i}^{\prime}, \lambda_{j}^{\prime}\right)$ such as $\hat{\nu}_{i}^{\prime}>\hat{\nu}_{i}$ and $\hat{\nu}_{j}^{\prime} \geq \hat{\nu}_{j}$, or $\hat{\nu}_{i}^{\prime} \geq \hat{\nu}_{i}$ and $\hat{\nu}_{j}^{\prime}>\hat{\nu}_{j}$. Therefore $\left(\hat{\nu}_{i}, \hat{\nu}_{j}\right)$ are Pareto optimal payoffs. 
Corollary. $\left(\hat{\nu}_{i}, \hat{\nu}_{j}\right)$ are Pareto optimal payoffs if $\lambda_{i}=1$ and $\lambda_{j} \in[0,1]$ or $\lambda_{i} \in$ $[0,1]$ and $\lambda_{j}=1$.

When $\lambda_{i}=1$ and $\lambda_{j} \in[0,1]$ :

$$
\begin{aligned}
\hat{\nu}_{i} & =G_{P}+\lambda_{j} G_{S} \\
\hat{\nu}_{j} & =\lambda_{j} G_{P}+\left(1-\lambda_{j}\right) G_{B}+G_{S}
\end{aligned}
$$

By combining Equations (A7) and (A8):

$$
\hat{\nu}_{i}=\frac{G_{S}}{G_{P}-G_{B}} \hat{\nu}_{j}+\frac{\left(G_{P}-G_{B}-G_{S}\right)\left(G_{P}+G_{S}\right)}{G_{P}-G_{B}}
$$

When $\lambda_{i} \in[0,1]$ and $\lambda_{j}=1$ :

$$
\begin{aligned}
& \hat{\nu}_{i}=\lambda_{i} G_{P}+\left(1-\lambda_{i}\right) G_{B}+G_{S} \\
& \hat{\nu}_{j}=G_{P}+\lambda_{i} G_{S}
\end{aligned}
$$

By combining Equations (A10) and (A11):

$$
\hat{\nu}_{i}=\frac{G_{P}-G_{B}}{G_{S}} \hat{\nu}_{j}+\frac{\left(G_{P}-G_{B}-G_{S}\right)\left(G_{P}+G_{S}\right)}{-G_{S}}
$$

When $\lambda_{i}=1$ and $\lambda_{j}=0, \hat{\nu}_{j}=G_{B}+G_{S}$. When $\lambda_{i}=1$ and $\lambda_{j}=1, \hat{\nu}_{j}=$ $G_{P}+G_{S}$. When $\lambda_{i}=0$ and $\lambda_{j}=1, \hat{\nu}_{j}=G_{P}$. Thus:

$$
\begin{cases}\hat{\nu}_{i}=\frac{G_{S}}{G_{P}-G_{B}} \hat{\nu}_{j}+\frac{\left(G_{P}-G_{B}-G_{S}\right)\left(G_{P}+G_{S}\right)}{G_{P}-G_{B}} & \forall \hat{\nu}_{j} \in\left[G_{B}+G_{S}, G_{P}+G_{S}\right] \\ \hat{\nu}_{i}=\frac{G_{P}-G_{B}}{G_{S}} \hat{\nu}_{j}+\frac{\left(G_{P}-G_{B}-G_{S}\right)\left(G_{P}+G_{S}\right)}{-G_{S}} & \forall \hat{\nu}_{j} \in\left[G_{P}+G_{S}, G_{P}\right]\end{cases}
$$

\section{Proof of Proposition 13}

Pareto Optimal SPNE. This proposition is a combination of Propositions 10, 11 and 12. Proposition 12 shows that all payoffs $\left(\hat{\nu}_{i}, \hat{\nu}_{j}\right)$ are Pareto optimal payoffs as long as the strategies $\left(\sigma_{i}, \sigma_{j}\right)$ supporting them are such that $\lambda_{i}=1$ and $\lambda_{j} \in[0,1]$ or $\lambda_{i} \in[0,1]$ and $\lambda_{j}=1$.

However, some of these pairs of payoffs $\left(\hat{\nu}_{i}, \hat{\nu}_{j}\right)$ give for one of the players a payoff smaller than the minmax payoff $G_{B}$. According to Proposition 10, these pairs of payoff are not supportable as average discounted payoffs of a subgame perfect Nash equilibria.

Proposition 11 shows that the minimum supportable payoff for player $i$ is reached when $\lambda_{j}=1$ and $\lambda_{i}=\lambda^{*}$. Hence, all the payoffs involving a pair of mixed strategies $\left(\lambda_{i}, \lambda_{j}\right)$ such that $\lambda_{i}=1$ and $\lambda_{j} \in\left[0, \lambda^{*}\left[\right.\right.$ or $\lambda_{i} \in\left[0, \lambda^{*}[\right.$ and $\lambda_{j}=1$ are Pareto optimal but are not supportable as SPNE payoffs.

Therefore only the Pareto optimal payoff brought by the pair of strategies $\left(\sigma_{i}^{*}, \sigma_{j}^{*}\right)$ such as $\lambda_{i}=1$ and $\lambda_{j} \in\left[\lambda^{*}, 1\right]$ or $\lambda_{i} \in\left[\lambda^{*}, 1\right]$ and $\lambda_{j}=1$ are supportable as average discounted subgame perfect Nash equilibrium payoffs. As a consequence, all the subgame Nash equilibria based on the strategies $\left(\sigma_{i}^{*}, \sigma_{j}^{*}\right)$ as defined above are Pareto optimal equilibria. 
Corollary (1). This corollary is a combination of the results of the corollary of Proposition 12 and of Proposition 13. The same methods used for the proof of the corollary of Proposition 12 is applied. In addition, the restrictions imposed on $\lambda_{i}$ in Proposition 13 are used. Thus the lowest value for $\hat{\nu}_{i}$ is $G_{B}$ and the highest value is $\hat{\nu}_{i}^{\max }$, as defined in Proposition 11.

Therefore, a subgame perfect Nash equilibrium based on the strategy profiles $\left(\sigma_{i}^{*}, \sigma_{j}^{*}\right)$ is Pareto optimal if an only if the equilibrium average discounted payoffs $\left(\hat{\nu}_{i}, \hat{\nu}_{j}\right)$ are such as:

$\begin{cases}\hat{\nu}_{i}=\frac{G_{S}}{G_{P}-G_{B}} \hat{\nu}_{j}+\frac{\left(G_{P}-G_{B}-G_{S}\right)\left(G_{P}+G_{S}\right)}{G_{P}-G_{B}} & \forall \hat{\nu}_{j} \in\left[G_{B}, G_{P}+G_{S}\right] \\ \hat{\nu}_{i}=\frac{G_{P}-G_{B}}{G_{S}} \hat{\nu}_{j}+\frac{\left(G_{P}-G_{B}-G_{S}\right)\left(G_{P}+G_{S}\right)}{-G_{S}} & \forall \hat{\nu}_{j} \in\left[G_{P}+G_{S}, G_{P}-\frac{G_{S}{ }^{2}}{G_{P}-G_{B}}\right]\end{cases}$

Corollary (2) Pareto Perfection and Renegotiation-Proofness. A renegotiationproof equilibrium is defined by Fudenberg and Tirole (1993) as an equilibrium that can not be renegotiated by any of the players. Renegotiation means that a player decides to play temporarily the WSPE in order to get another equilibrium, in which all the players would be better off, to be adopted. A renegotiation-proof equilibrium is also called "Pareto perfect" as it is not Pareto dominated by any other equilibria.

Two forms of renegotiation-proofness are discussed in the literature. Farrell and Maskin (1989) introduce the concept of weakly renegotiation-proof (WRP) equilibria which tests the internal Pareto consistency - e.g. none of the continuation payoffs of the strategies used in the equilibrium should be dominated by any of the continuation payoffs of another equilibrium. However the WRP concept, although useful for some games, leads to some problems in the case of repeated games where non-optimal stage-game dominant strategies exist ${ }^{29}$.

Pearce (1987), on the contrary, develops an alternative concept of renegotiation proofness based on the external Pareto consistency. This means that an equilibrium is renegotiation-proof if none of its continuation payoffs are dominated by all the continuation payoffs of another equilibrium. This is the concept of renegotiation-proofness we decided to use in this article.

Let $\left(\sigma_{i}^{*}, \sigma_{j}^{*}\right)$ be a Pareto optimal SPNE as defined in Proposition 13. The strategy profile $\sigma_{i}^{*}$ is based on the mixed strategy $\lambda_{i}^{*}$, where $P S$ is played with probability $\lambda_{i}^{*}$ and $\bar{P} S$ with probability $1-\lambda_{i}^{*}$, with a minmax payoff based grim-trigger punishment:

$$
\sigma_{i}^{*}=\left\{\begin{array}{l}
\text { Play } \lambda_{i}^{*} \text { if }\left(\lambda_{i}^{*}, \lambda_{j}^{*}\right) \text { was played during the precedent stage-game } \\
\text { Play } \bar{P} \bar{S} \text { otherwise }
\end{array}\right.
$$

All the punishment continuation payoffs of $\left(\sigma_{i}^{*}, \sigma_{j}^{*}\right)$ are dominated by the cooperative continuation payoffs of other cooperative equilibria, since the punishment continuation payoffs are those of the $\mathrm{WSPE}^{30}$. However, as by assumption $\lambda_{i}^{*}=1$ and $\lambda_{j}^{*} \in\left[\lambda^{*}, 1\right]$ or $\lambda_{i}^{*} \in\left[\lambda^{*}, 1\right]$ and $\lambda_{j}^{*}=1$, there are no equilibria involving continuation payoffs higher for both of the players than the cooperative continuation payoffs - based on the pair $\left(\lambda_{i}^{*}, \lambda_{j}^{*}\right)$ - of the $\left(\sigma_{i}^{*}, \sigma_{j}^{*}\right)$ equilibrium $^{31}$.

\footnotetext{
${ }^{29}$ See discussion in Fudenberg and Tirole (1993).

${ }^{30}$ Hence, this equilibrium does not satisfy the weakly renegotiation-proof condition.

${ }^{31}$ See proof of Proposition 12.
} 
Therefore the $\left(\sigma_{i}^{*}, \sigma_{j}^{*}\right)$ equilibrium is externally renegotiation-proof and is Pareto perfect.

\section{Proof of Proposition 14}

Impact of Rivalness.

$$
\frac{\partial\left(G_{P}+G_{S}\right)}{\partial R}=-(1-E)\left(1+N_{i}\right) u_{i}
$$

Thus for all $E \in[0,1], N_{i} \in[0,1]$ and $u_{i} \geq 0$ :

$$
\frac{\partial\left(G_{P}+G_{S}\right)}{\partial R} \leq 0
$$

Impact of excludability.

$$
\frac{\partial\left(G_{P}+G_{S}\right)}{\partial E}=R u_{i}\left(N_{i}+1\right)-N_{i} u_{i}
$$

Thus for all $R \in[0,1], N_{i} \in[0,1]$ and $u_{i} \geq 0$ :

$$
\frac{\partial\left(G_{P}+G_{S}\right)}{\partial E}<0 \Leftrightarrow R<\frac{N_{i}}{N_{i}+1}
$$

And:

$$
\frac{\partial\left(G_{P}+G_{S}\right)}{\partial E}>0 \Leftrightarrow R>\frac{N_{i}}{N_{i}+1}
$$

